\title{
AN OVERVIEW OF MICROWAVE RESONANCE THERAPY AND EEG CORRELATES OF MICROWAVE RESONANCE RELAXATION AND OTHER CONSCIOUSNESS ALTERING TECHNIQUES
}

\author{
D. Raković, ${ }^{1,2}$ Z. Jovanović-Ignjatić, ${ }^{2}$ D. Radenović ${ }^{1,2}$, M. Tomašević ${ }^{2,3}$, E. Jovanov ${ }^{1,2,4}$, \\ V. Radivojević $c^{2,5}, \breve{Z}$. Martinović ${ }^{2,5}$, P. Šuković ${ }^{1}$, M. Car $^{6}$, and L. Škarić ${ }^{1}$ \\ ${ }^{1}$ Faculty of Electrical Engineering, PO Box 35-54, 11120 Belgrade, Yugoslavia \\ E-mail: rakovic@net.yu \\ ${ }^{2}$ International Anti - Stress Center (IASC), Smiljanićeva 11, Belgrade, Yugoslavia \\ 3 "Vinča" Institute of Nuclear Sciences, Belgrade, Yugoslavia \\ ${ }^{4}$ Institute "M. Pupin", Computer System Department, PO Box 15, Belgrade, Yugoslavia \\ ${ }^{5}$ Institute for Mental Health, Department of Clinical Neurophysiology, \\ Palmotićeva 37, Belgrade, Yugoslavia \\ ${ }^{6}$ Institute for Biological Research, Center for Multidisciplinary Studies, \\ 29. Novembra 142, Belgrade, Yugoslavia
}

\begin{abstract}
Microwave Resonance Therapy (MRT) is a novel medical treatment, which represents a synthesis of the ancient Chinese traditional knowledge in medicine (acupuncture) and recent breakthroughs in biophysics. Affecting the appropriate acupuncture points by the generation of high frequency microwaves $(52-78 \mathrm{GHz})$, remarkable clinical results of the treatment are being achieved. In this paper the quantum-like macroscopic biophysical bases of the MRT are elaborated too, offering a new insight in the mechanisms of the assembling gap junction hemichannels upon the internal microwave (MW) electromagnetic field spatio-temporal maximums at the temporary position of the acupuncture system, and hence the very biophysical nature of the temporary psychosomatic health or disease. Such a picture also supports the EM/ionic "optical" ultralowfrequency modulated MW quantum holographic neural network-like function of the acupuncture system (similar to complex-valued oscillatory holographic Hopfield-like neural networks), and its essential relation to consciousness, as strongly suggested from biophysical modeling of altered states of consciousness. Finally, our electroencephalographic investigations showed more or less characteristic EEG features in many subjects of relaxation induced by microwave resonance therapy applied to corresponding acupuncture points, as well as by some other activities which may alter consciousness (transcendental meditation, musicogenic states, healer/healee noncontact interaction, drowsiness), demonstrating that EEG may permit rapid and reproducible identification of different states of consciousness, useful in evaluating the effectiveness of consciousness altering techniques and related stress reduction.
\end{abstract}

Keywords: acupuncture, microwave resonance therapy (MRT), biophysical basis, EEG correlates, microwave resonance relaxation, some other consciousness altering techniques (transcendental meditation, musicogenic states, healer/healee interaction, alert wakefulness/drowsy wakefulness).

In spite of significant financial and human resources engaged in biomedical investigations and health prophylaxis, human health is still jeopardized by numerous modern psychosomatic diseases, having its convenient ground in modern men exposed to everyday stress. Since 
conventional partial methods have failed to prevent and treat these health problems, new approaches are required oriented in healing a man as a whole (not a disease which is only a symptom of the disordered wholeness), which will include holistic traditional methods, focused on human acupuncture system and consciousness as significant psychosomatic global regulators of health, but supported with contemporary biomedical technologies.

Therefore, in this paper an overview of Microwave Resonance Therapy (MRT, as a synthesis of the ancient acupuncture traditional medicine and recent breakthroughs in biophysics), together with electroencephalographic (EEG) correlates of MRT relaxation (and some other consciousness altering techniques too), will be presented.

\section{BIOPHYSICAL BASES OF TRADITIONAL MEDICINE}

The yin-yang concept of acupuncture, as a network of energy-information processes of an organism, originating from embryological development, represents one of the most specific and most useful segments of the renowned Chinese traditional medicine [1-3]. Indian traditional medicine, and especially one of its most prominent representatives, swara yoga, is also acquainted with an energy system analogous to the Chinese acupuncture system: in Indian terminology the qi is known as prana, and meridians as nadis (14 of them being basic, like in acupuncture, although three of them being of special medical and spiritual significance: ida, pingala, and shushumna) [4].

Besides its practical medical aspects, Chinese traditional medicine is deeply colored with mystical connotations, which is one of the reasons why Western science has been reluctant to accept experiences of Eastern tradition. Another reason is that within the 12 visceral organs corresponding to the 12 paired meridians, Chinese tradition has not included the brain and endocrine glands: however, in the past few decades it was found that the acupuncture system was in close functional interaction with both the central nervous system and endocrine system, as well as with peripheral and autonomic nervous systems [1-3]. The final reason was a lack of clear anatomical basis of the Chinese acupuncture system: however, some investigations of gap junctions (specific evolutionary older type of intercellular channels, transporting small metabolites and secondary messengers between inexcitable cells, and also small ions between excitable cells of the cardiac muscle, smooth muscle, epithelial liver cells, neurons with electrical synapses [5]) have shown their increased concentration inside the acupuncture points and meridians for an order of magnitude [6], and their role in epithelial conception of genesis of the system of vital meridians and their acupuncture points [7]. All gap junctions consist of a pair of cylinders (connexons), one in the presynaptic and the other in the postsynaptic cell, which meet in the gap between the two membranes and contact, by means of homophilic (cell specific) interactions, to establish a communicating channel between the cytoplasm of the two cells; each cylindrical connexon is made of six identical protein subunits (connexins) - which have a function to selfassemble connexon hemi-channels and to recognize its counterpart hemi-channel and complete a conductive channel. The whole such process is very flexible and depends on the electrochemical potential of the cell: the very conductivity of the gap junction can be modulated by intracellular $\mathrm{pH}$-factor, $\mathrm{Ca}^{2+}$-ions, neurotransmitters and second messengers, and even by voltage - which can change the conformational state of the connexon (like the shutter in a camera) [5,8].

A better organization of cell structures and an ionic basis of the qi entity of the acupuncture system is also suggested by an order of magnitude higher skin electrical conductivity of the acupuncture points in respect to the surrounding tissue, as well as much higher reabsorption of the air ions in these points [2] - implying higher concentration of the ions and gap junctions in the acupuncture points and meridians too.

Ionic acupuncture currents, and accompanying electromagnetic (EM) fields, have ultralowfrequency (ULF) and microwave (MW) components, i.e. the very fast MW component is amplitude-modulated by significantly slower ULF component [9,10], this being in overall agreement with the frequency and power windowing in tissue interactions with weak electromagnetic fields [11]. In support of the ULF nature of ionic currents in acupuncture channels, 
one can cite the resonance ULF stimulation of the acupuncture analgesia endorfin (at $\sim 4 \mathrm{~Hz}$ ) and serotonine and/or norepinerphine (at $\sim 200 \mathrm{~Hz}$ ) mechanisms [12], as well as the efficiency of the German school of the resonance ULF therapy [13]. On the other hand, the evidence for the MW component of ionic acupuncture currents is provided by the efficiency of the Ukrainian-Russian school of the resonance MW $(\sim 30-300 \mathrm{GHz})$ therapy $[14,15]$.

Theoretical predictions of the biophysical model of altered and transitional states of consciousness $[9,10]$ also imply an ionic nature of the qi entity of the Chinese acupuncture system, with possibility of its partial displacements from the skin surface in these states; in this context, the displaced part of ionic acupuncture structure would function as an "optical" MW regulatory neural network, modulated by ULF brainwave information and/or visual information perceived directly by the partially displaced ionic system, having also the sensory role in altered states of consciousness [16]. In the framework of the model, the qi has its interpretation in ions, out of which negative ions having anabolic influence flowing predominantly through the left circulatory part of the acupuncture system, while the positive ions having catabolic influence flowing predominantly through the right circulatory part of the acupuncture system (cf. also footnote 2), with informational contents coded in the form of spatio-temporal distributions of the acupuncture ionic currents and accompanying MW and ULF EM fields.

This is in accordance with the enhanced cell growth toward (negative) cathode and reduced cell growth toward (positive) anode in small continuous, pulsed or focal electric fields [19,20], in consistence with the model that the mechanism underlaying acupuncture is similar to that of growth control, acupuncture points being the organizing centers in morphogenesis [21]. Such an interpretation is additionally supported by electropuncture therapy, where negative pulse stimulation of a point tonifies its corresponding function while positive pulse stimulation sedates the function [22], implying that a tonification of the organ is related with its regenerative anabolic yin overall function while the sedation is related with its degradative catabolic yang overall function. Therefore, the role of acupuncture stimulation might be the balancing of activity of the positive and negative ions within the body, corresponding to healthy functioning (cf. also [27]). Besides, it seems that the external qi gong treatment [23] or healing process [24] might be related with the ionic diffusion between the healer and healee and/or information transfer of the MW and ULF EM patterns responsible for normal functioning of acupuncture system and overall health $[9,10]$.

This also implies the significance of aeroionic balance $[9,10,25,26]$ and regular rhythmical breathing, recognized in Indian tradition. According to swara yoga [4] the rhythmical breathing through nose is especially important, in order to inhale as many as possible aeroions (prana) within the two (out of three) most significant nadis (the left ida and the right pingala, with their entrances in corresponding nostrils) [27]: this is recommended in fresh and nonpoluted air, when even some excess of negative ions exists, with the relaxing healthy influence on the body (in the same context, in closed environments a microclimate engineering can be recommended by applying aeroionizers which produce an excess of negative ions [25]). It should be added that swara yoga is also quite acquainted with the significance of $\sim 2$ hour (brain and nasal) ultradian rhythms, and recommends their simple nasal control in the process of recovering or keeping energy and emotional balance of the organism [4].

\section{AN OVERVIEW OF MICROWAVE RESONANCE THERAPY}

The application of low-intensity EM MW fields (of intensity range $\sim 10^{-3}-10^{-9} \mathrm{~W} / \mathrm{cm}^{2}$, in extremely high frequency (EHF) range $\sim 30-300 \mathrm{GHz}$, and millimeter (MM) wavelengths range $1-10 \mathrm{~mm}$ ) in biomedicine is a new trend, originated in former USSR in mid-1960's with the advent of unique broadband backward-wave tube oscillators by two radioelectronics groups of USSR Academy of Sciences in Moscow and Kharkov: the influence of EM MW radiation upon nonhuman biological objects appeared shortly afterwards in Zalubovskaya's dissertation in Kharkov [29] and publication of Devyatkov's Moscow group [30]. However, the necessity for application of 
microwave resonance therapy (MRT) upon acupuncture points was discovered in the early 1980's by Kiev scientists Andreev, Bely, and Si'tko as appearance of sharply-resonant characteristic eigenfrequencies of human organism [31], stimulated by previous empirical MW "side effects" observed by Odessa physicians Cherkasov and Nedzveckii [32]. Subsequently, MRT (EHF) therapy has made successful headway in former USSR (especially in Ukraina and Russia, at present, with more than 500.000 successfully treated patients of different pathologies in more than 1500 MRT cabinets), becoming a new powerful therapeutic method, internationally introduced by Sit'ko in 1990, when he was awarded a gold medal in Brussels at the 39th Annual World Exposition of Innovations, Research and New Technologies, and shortly afterwards the MRT was introduced worldwide [33].

But at present, despite the successes in medical practice, the physical mechanisms of interaction of EM MW radiation and biological organisms are not quite clear, due to several peculiarities of the MRT method [14]: (1) sharply-resonant sensory response of the disordered organism to small changes of the external MW radiation frequency $(0.01-0.1 \%)$; (2) low-intensity (down to $10^{-9} \mathrm{~W} / \mathrm{cm}^{2}$ ) and low-energy (down to $10^{-4} \mathrm{eV}$ ) of the biologically efficient MW radiation rather below the thermal noise effect $\left(\sim 10^{-2} \mathrm{~W} / \mathrm{cm}^{2}\right.$ and $\left.\sim 10^{-2} \mathrm{eV}\right)$; and (3) negligible MW energy losses at its propagation for significant distances down acupuncture meridians $(\sim 1 \mathrm{~m})$ from the exposed acupuncture points. Such quantum-like coherent characteristics of MRT inspired Sit'ko and coworkers to propose a quantum physics of alive [34], based on nonequilibrium thermodynamics and nonlinear electrodynamics of novel dynamically stable spatio-temporal biological structures as a macroscopic quantum systems with nonlocal selfconsistent macroscopic quantum potentials, which can give rise to nonlinear coherent EM MW long-range maser-like excitations of biological nonlinear absorption medium with the cells as active centers. In the framework of this model, acupuncture meridians might be related to eigenfrequences and spatio-temporal eigenwaves distributions of every biological quantum system, being its individual characteristics.

The above quantum picture can be more simply visualized [10] considering acupuncture system as a dynamic structure assembled at the locations of maximums of the interference threedimensional (3D) standing waves resulted in reflections from the skin of the nonlinear coherent EM MW Fröhlich excitations of strongly polarized molecular subunits in the cell membranes and cytoplasmatic proteins [35], supported also by other investigations which suggest that formation of gap junctions (of higher density at acupuncture points and meridians [6]) involves a gating process as well as assembly of connexon hemichannels (or even connexin monomers) which reside in the membrane prior to joining with a counterpart in an opposite cell [8], which might be stimulated at spatio-temporal maximums of MW EM field of the organism (similarly to voltage-sensitive conductivity of gap-junctions [5]).

In that context the explanation for the efficiency of the MRT, as a noninvasive biophysical medical treatment, should be sought [10]: some disorders in the organism (related to local changes of dielectric properties of tissues and organs) give rise to deformation in the standing wave structure of the electrical field of the MW EM field of the organism, which influences corresponding changes in the spatio-temporal structure of the acupuncture system, and consequently resonance frequencies of its meridians, resulting in some disease. During the therapy, applying the MW source at a corresponding acupuncture point the excited meridian of patient's acupuncture system relaxes to the previous healthy condition, while reaching its normal resonance frequency responses upon the wide spectrum MW source - and following physiological mechanisms of acupuncture regulation (via nervous and endocrine systems [1-3]) the organism biochemically overcomes the disease.

The afore-mentioned resonance frequency changes can be monitored indirectly by searching for the MW resonance frequency of the meridian before and during the MRT treatment, using coherent MW generators with manually changeable frequency [33]: it should be noted that sensory response to (gradually changing) resonance frequency decreases during recovering, practically vanishing when regaining normal healthy condition. This suggests that healthy condition might be considered as an absolute minimum (ground state) of Sit'ko's nonlocal selfconsistent macroscopic quantum potential of the organism, some disorders of an acupuncture system 
corresponding to higher minimums of the (spatio-temporally changeable) potential hypersurface in energy-configuration space, which possibly explains the higher sensory responses of the more excited (more disordered) acupuncture system, and poor MRT sensory response of the healthy acupuncture system being already in the ground state (cf. Fig. 1).

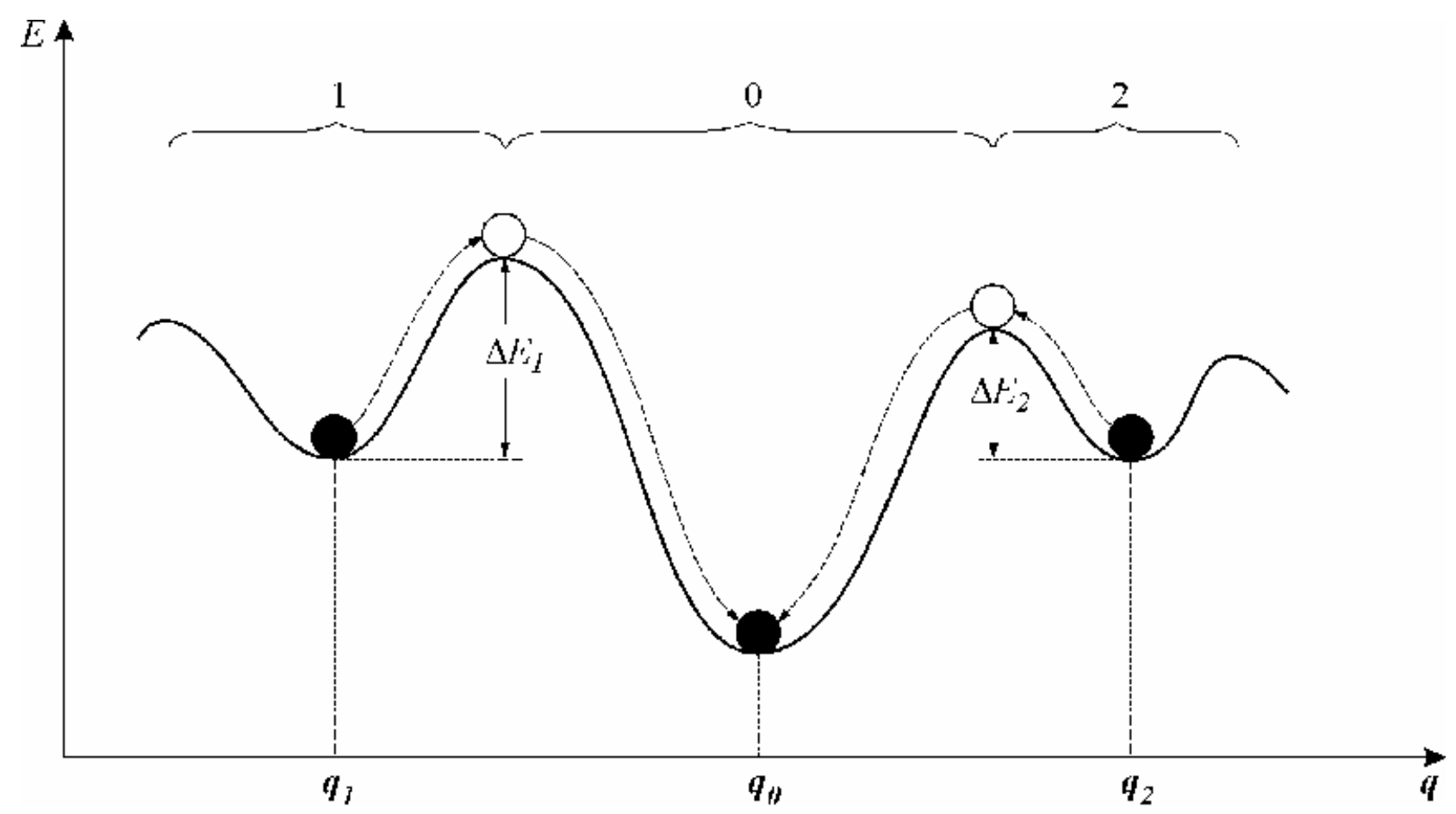

Figure 1. Schematic presentation of the potential hypersurface of nonlocal selfconsistent macroscopic quantum potential of the acupuncture system of an organism in energy-configuration $(E, \boldsymbol{q})$ space: 0 - basin of attraction of the ground (healthy) state $\boldsymbol{q}_{0} ; 1$ and 2 - basins of attraction of the two excited (disordered) states $\boldsymbol{q}_{1}$ and $\boldsymbol{q}_{2}$. It should be poined out that energy surface changes gradually during the MRT treatment, by decreasing the potential barriers $\Delta E_{1}$ and $\Delta E_{2}$ (and corresponding MRT sensory responses) of the potential wells 1 or 2 , to be overcome in traversing to the 0 basin of attraction of the ground (healthy) state, when organism gradually recovers by further spontaneous approaching the healthy state $\boldsymbol{q}_{\boldsymbol{0}}$ (with poor MRT sensory response, as already being in the ground-state). Such a picture is very close to the associative neural networks ones in their energy-configuration spaces, and to pattern recognition as convergence of the neural networks to the bottoms of the potential hypersurfaces, being the attractors of neural networks memory patterns $\boldsymbol{q}_{0}, \boldsymbol{q}_{1}, \boldsymbol{q}_{2}$ [37].

Both the coherent and noise spectrum MW generators can be used in therapeutic practice [33]. The coherent spectrum MW generators with manually changeable frequency (from 52 to 70 $\mathrm{GHz}$ ) are far less suitable in practice, because of much longer seeking of the resonant frequency, dependent on individual properties of the organism and the subjective state of the patient, which can result in therapeutic mistakes and overdosing. The noise spectrum MW generators enable simultaneous excitation of all possibly therapeutic resonance $\mathrm{MW}$ frequencies $(52-78 \mathrm{GHz})$, and an organism continuously resonantly responds to currently appropriate (and changeable during therapy) frequency. Up to $10 \mathrm{~mW}$ low-power microwave generators, of the output power density of $0.2-5 \mu \mathrm{W} / \mathrm{cm}^{2}$ - much lower than biologically limited $10 \mathrm{~mW} / \mathrm{cm}^{2}$ during 8 hours, as prescribed by USA National Standards, or $10 \mu \mathrm{W} / \mathrm{cm}^{2}$ during 8 hours, as prescribed by Russian and Ukrainian National Standards [33] - are power supplied by the $220 \pm 22 \mathrm{~V} / 50 \mathrm{~Hz}$ a.c.

The output power density as well as the duration of the treatment significantly influence the MW absorbed dose and corresponding MRT bioeffects, which can be biostimulative for low-level therapeutically recommended doses of typical 20-minute daily MRT treatments (causing local temperature increases up to $38{ }^{\circ} \mathrm{C}$, with maximally fast bioeffect), and biodepressive and even biodestructive effects for much higher doses (causing much higher and harmful local temperature 
increases) [33]. For an individual MW dose prescription, the monitoring of the level of mitochondrial Succinate Dehydrogenase (SDG) enzyme in lymphocytes of peripheral blood was suggested by Sit'ko's group [33], because the metabolism of lymphocytes can be used as the sickness degree index: it has been demonstrated that the lymphocyte system "remembers" a former state of health, has the information about the present one, and points out the succeeding changes that can occur in the further development of the illness [36]; the Sit'ko's group did not refer to a type of lymphocytes, save for the case of oncological patients when they specified $\mathrm{T}$ cells. The level of the SDG enzyme is estimated microscopically by enumerating the granules of formazan (the product of biochemical reaction of the SDG with the substrate) within the smeared and colored blood specimen.

The mobility of the MRT apparatuses (with the exception of the stationary generator) enables their use in various conditions, beginning with home conditions when the doctor comes to the house of the patient, then through emergency and urgent help, all the way to stationary institutions (physiotherapeutic cabinets, climate sanatoriums, coronary units, orthopedic-surgical and neurological institutions).

The recommended MRT treatment [33] at an acupuncture point is maximum 10 minutes, while the duration of the whole treatment is maximum 20 minutes, applied daily. The 10 treatments in a course are applied (except in very difficult stadiums of diseases when they have to be prolonged), with minimum pause of 21 days and optimal of one month, in the case a repeated course is necessary. The specific course of prophylaxis is advised at every 6 months.

The evaluation of the clinical and economical efficiency in the former USSR during almost one decade included more than 500,000 patients with over 60 different pathologies taken in tens of referent institutions [14,33]: (a) the results of the MRT research showed high treatment efficiency (60-95\%), depending on the kind of disease, state of the evolved pathological processes, and individual differences; and (b) the healing process is shortened 1.5-2 times, and there are no significant side effects (save for possible transient negative and uncomfortable general reactions, stated below). So, the high clinical and economical efficiency of MRT can be pointed out. Our experience [10,44] during the six year period 1993-98, based on medical practice with several hundreds patients, confirmed the high effectiveness of MRT, based on relief of symptoms, prolonged remissions, and referent diagnostic procedures.

A list of diseases which can be treated by MRT is still open, being continuously extended. To date, MRT has been indicated [33] in: (1) surgery, (2) orthopedic and traumatology, (3) neurology, (4) psychiatry, (5) addiction, (6) cardiovascular disorders, (7) urology, (8) gynecology, (9) gastroenterology, (10) upper respiratory tract, (11) pulmology, (12) dermatology, (13) allergology, (14) immunology, and (15) oncology.

In the moment of establishing the resonant frequency the patient's sensory response can be manifested as local, system (related to organ or system), and general (only in a few percentage of the patients the sensory response was missing $[33,43]$ ).

Local reaction appears in $74 \%$ of the patients [33], as a feeling of warmth, pricking, weight, goosflesh, bites, or cold at the contact point. System reaction appears in 97\% of the patients [33]. General reaction appears in $32 \%$ of the patients [33] in the form of comfortable and uncomfortable reactions. Comfortable reactions appear in the form of quiet colors in front of the sight (green, blue), feeling of warmth within the body, drowsiness, feeling of pleasant relaxation and weakness in muscles, ease within entire body, which can be registered in the change of blood pressure, pulse, ECG and EEG rhythms, and respiratory normalization [10]. Uncomfortable reactions can appear sometimes when colors begin to run away or when one sees bright colors or feels suffocation, when further MRT stimulation should be terminated, and continued later on but with lower dose. Transient negative reactions might appear sometimes in the form of the symptom enhancement, followed by positive response [33]. That transient aggravation might appear at the very beginning of the treatment, or after 2-3 days, or in the middle of the treatment. Nevertheless, after 2-3 days most of the patients react with general emotional and biophysical enthusiasm. 


\section{EEG CORRELATES OF MICROWAVE RESONANCE RELAXATION AND OTHER CONSCIOUSNESS ALTERING TECHNIQUES}

This was our motivation to start examination of EEG correlates of microwave resonance relaxation and other consciousness altering techniques (transcendental meditation, musicogenic states, healer/healee noncontact interaction, and drowsiness) [45] - motivated also by search for rapid and reproducible EEG identification of different states of consciousness, useful in evaluating the effectiveness of consciousness altering techniques and related stress reduction.

\section{Microwave resonance relaxation}

The MRT was applied by the wide spectrum apparatus POROG-3, and the measurement of frequency was achieved by the narrow spectrum apparatus AMRT-01, adjusted manually. The frequency range of the POROG-3 is $52-78 \mathrm{GHz}$, with the output power density of $0.2-5 \mu \mathrm{W} / \mathrm{cm}^{2}$. MRT generator was applied on acupuncture points in following order: $\mathrm{Du} 20$, and the left-side points Li 4, Pc 6, H 7 and Ap 55, which resulted in relaxation, similarly to the parasympathicus effect [1-3]. The choice of the acupuncture points for the relaxation seance was achieved on the basis of well known principles of acupuncture stimulation, characteristics of the chosen points, and the therapeutist experience.

The study was carried out on 28 healthy adult volunteers (13 males and 15 females).

The experiment was conducted in a soundproof room, dimly lit for observation. Subjects were laid comfortably. Each session was divided into three sequential periods: (1) relaxing 5 min with eyes closed; (2) MRT $20 \mathrm{~min}$; and (3) relaxing $5 \mathrm{~min}$ with eyes closed. During the first and third periods two random samples, one minute each, were recorded for every subjects. The EEG records were stored on a hard disk.

The subjects were classified in two groups: the group 1 (11 subjects) not previously subjected to the MRT treatment, and the group 2 (17 subjects) being subjected to the MRT in the past two years.

Both groups of subjects have significant changes in the EEG power over the whole head in the $\alpha$ and $\beta_{1}$ frequency bands, with observation that a percentage of subjects with minor reactions is much less in the group 1. The group 2 has also the significant EEG power changes over the whole head in a $\delta$ frequency range.

Two examples of spectral arrays in our subjects $\left(\mathrm{N}^{\mathrm{O}} \mathrm{s} 7\right.$ and 18), showing the decrease in the EEG power, are shown in Fig. 2(a, b).

Within the whole frequency range (1-30 Hz), 37 channels in the first group and 22 channels in the second group with the power changes in more than $50 \%$ subjects are notified. The changes are evident in $\delta, \alpha$, and $\beta_{1}$ frequency bands. The most prominent power changes in all frequency bands are observed in the channels 3 (T3), 11 (C3), and 12 (C4) of the group 1, and in the channels 6 (T6), 9 (F3), 11 (C3), and 13 (P3) of the group 2. The channels 3 (T3), 11 (C3), and $15(\mathrm{O} 1)$ of the group 1, and the channels 6 (T6) and 11 (C3) of the group 2 have power changes in more than 50\% subjects in 4 out of 5 frequency bands. In both groups of subjects, a decrease in the EEG power is more frequently observed than an increase. As an illustration, in Fig. 3 (a-c) the topographic mappings of the number of subjects (in \%) having the significant EEG power changes in the $\delta, \alpha$ and $\beta_{1}$ frequency bands, for the two groups of subjects are presented.

The changes in coherency are not too significant. Regarding the complete group of subjects, in $\delta$ frequency range the changes are observed in the backward temporal (T5 and T6), parietal (P3 and $\mathrm{P} 4$ ), and occipital (O1 and O2) regions in $25 \%, 25 \%$, and $42 \%$ of subjects, respectively. The changes in frontal region (F3 and F4) within $\alpha$ frequency band are observed in $29 \%$ of subjects, and in occipital region (O1 and $\mathrm{O} 2)$ within $\beta_{1}$ frequency band in $25 \%$ of subjects. Most prominent changes, over the whole frequency interval $(1-30 \mathrm{~Hz})$, are registered in occipital region (O1 and $\mathrm{O} 2)$. A decrease in the coherency is generally observed. 


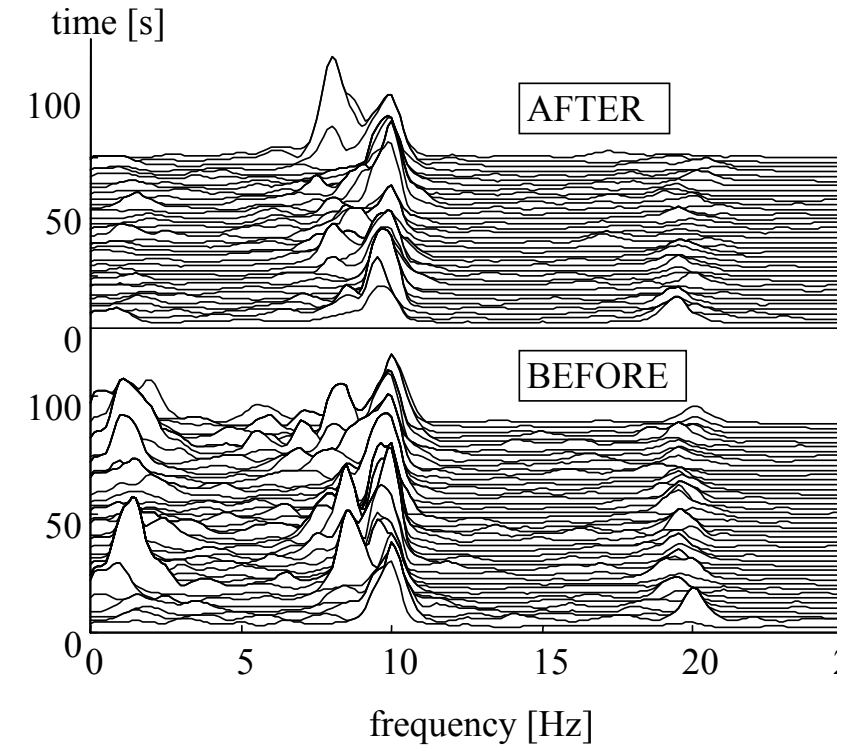

(a)

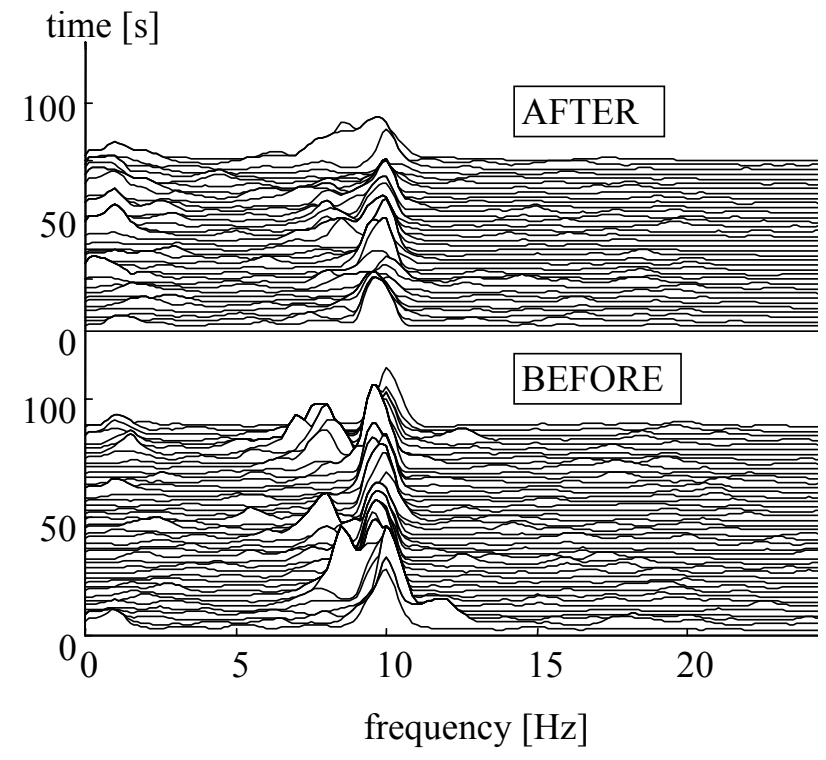

(b)

Figure 2. (a) Compressed power spectral arrays of the EEG for subject 7 obtained from the electrode C3 before and after MRT treatment, showing the decrease in the EEG power in all frequency bands; (b) Compressed power spectral arrays of the EEG for subject 18 obtained from the electrode T3 before and after MRT treatment, showing the decrease in the EEG power in $\alpha$ band.

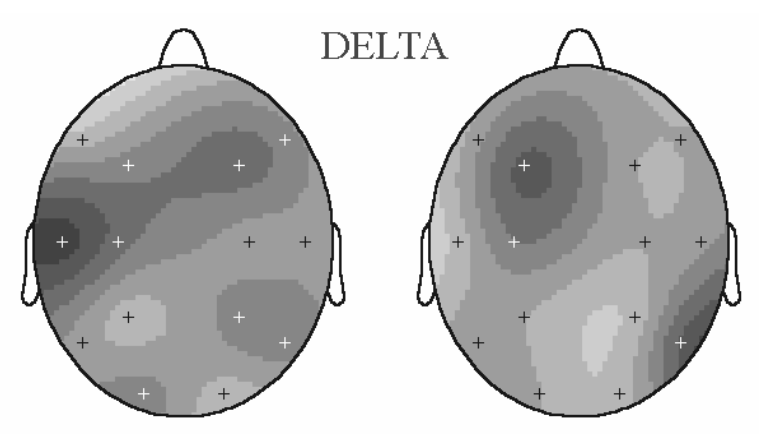

(a)

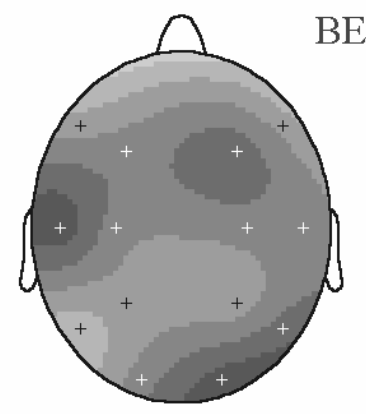

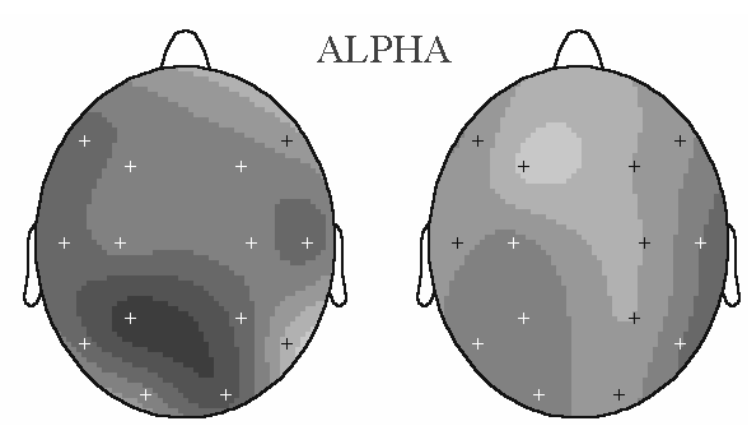

(b)

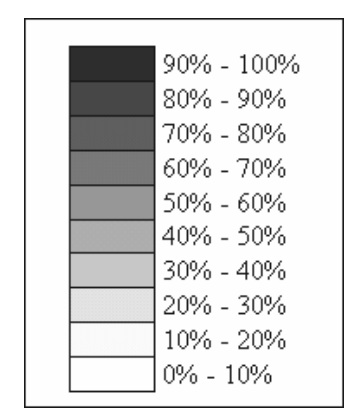

(c)

Figure 3. The topographic mappings of the number of subjects (in \%) of the group 1 (left) and the group 2 (right), having the significant EEG power changes in the: (a) $\delta$ band (1-4 Hz), (b) $\alpha$ band (8-13 Hz), and (c) $\beta_{1}$ band $(13-18 \mathrm{~Hz})$. The gradual percentage changes are presented in various degrees of shading, as designated in the insert. 


\section{Transcendental meditation}

In transcendental meditation (TM), normally practiced for periods of twenty minutes twice a day, the subject is instructed to sit quietly with eyes closed and is then taught to repeat a certain type of sound or "mantra" according to a particular definite set of instructions. The mantras are a set of short speech sounds, meaningless in themselves, preserved by an ancient vedic tradition and assigned to individuals by the instructor on the basis of a set of objective rules which is trained to apply. They are chosen so as to "resonate" with the structure of an individual nervous system.

Subjectively, the meditator usually reports an immediate sense of bodily quiet and relaxation. An important feature of the subjective experience of the TM technique is the "expansion" of consciousness [46]. As the mantra is experienced in successively finer stages, subjects report that the spatial extent of conscious self-awareness, which ordinarily seems to be localized in the area of head and upper body, undergoes a progressive expansion.

Wallace, Orme-Johnson, and Farrow [47], among others, have reported physiological changes during the practice of the TM technique that are consistent with these predictions, such as reduced oxygen uptake, reduced $\mathrm{CO}_{2}$ elimination, constant respiratory quotient, reduced respiratory minute ventilation, reduced respiratory frequency, reduced hearth rate, increased basal skin resistance, and EEG changes indicative of alertness. Wallace was led to propose that the TM technique produces a fourth major state of consciousness in which the mind remains alert while mental activity reaches a least excited state.

The study was carried out on 25 healthy adult volunteers who had been practicing the TM technique from 0.2 to 25 years, with a mean of 7.2 years. There were 15 males and 10 females, whose ages ranged from 17 to 68 years with a mean age of 32 years. All subjects were free of any medication. Prior to the experiment, subjects were informed verbally about all aspects of the experimental procedure.

The experiment was conducted in a soundproof room, dimly lit for observation. Subjects were seated comfortably. Each recording session was divided into two sequential periods: (1) relaxing $5 \mathrm{~min}$ with eyes closed and (2) meditating $15 \mathrm{~min}$. During those periods two random samples, one minute each, were recorded for every subject. The EEG record was stored on a hard disk.

The representative examples of spectral arrays in our subjects $\left(\mathrm{N}^{\mathrm{o}} \mathrm{s} 1\right.$ and 12) with slow $\alpha$ activity and $\theta$ burst during meditation, respectively, are shown in Fig. 4(a, b).

The comparison of the medians of partial EEG power for one derivation was performed using Wilcoxon matched pairs test. Fig. 5 shows the spatial distribution of the changes (z-scores) over the whole head. Shaded areas indicate the fields that have significant power increase. The primary sources of differences were the left frontal region (F3, $z=3.24, p=0.001)$ in $\theta$-band, right temporal region $(\mathrm{T} 4, z=2.65, p=0.008)$ in $\alpha_{1}$-band, left temporal region $(\mathrm{T} 3, z=2.73, p=0.006)$ in whole $\alpha$-band, and left prefrontal region (Fp1, $z=2.59, p=0,01)$ in $\beta_{1}$-band.

Spatial distributions of medians of correlation dimension over the whole head before and during meditation did not show statistically significant changes.

The present study confirms previous reports [47-51]. The meditators as a group displayed a significant increase of $\theta$ activity $(z=2.00, p=0.046)$ over the whole head. In particular, out of 25 meditators, $10(40 \%)$ significantly increased their $\theta$ activity during meditation, and $4(16 \%)$ significantly decreased. Analysis of each of the 16 derivations separately showed that the prominent $\theta$ wave activity is present in the frontal, central, and right temporal regions at frequency of $8 \mathrm{~Hz}$ (channel F3, $z=3.91, p=0.0001$ ). The patterns of $\theta$ frequencies fluctuated. Observed hypersynchronous $\theta$ bursts were similar to the $\theta$ bursts occurring during phases of emotional excitation $[52,53]$.

The consistent changes in the other frequency bands were not observed in meditator group as a whole. Out of 25 subjects, $12(48 \%)$ significantly increased their slow $\alpha$ activity $(8-10.5 \mathrm{~Hz})$ 
during meditation, and $4(16 \%)$ significantly decreased. The prominent slow $\alpha$ activity occurred in right frontal, central and temporal regions. During TM, there was a significant increase of $\alpha$ activity in $13(52 \%)$ experimental subjects and significant decrease in $7(28 \%)$, most frequently in temporal region (T3-T4). The increase of slow $\alpha$ activity during the TM technique is apparently due to the nature of the technique, which according to adherents involves the increasingly abstract experience of quieter levels of mental activity, attained without concentration or procedures of controlling the mind [54]. Increased orderly functioning of the frontal and central regions of the brain may be correlated with this improvement in mental abilities, especially since these brain areas are known to be responsible for such activities as sensory-motor integration, memory, cognition, concentration, judgment, and volition [50]. Those changes may not necessarily occur in long-term meditators. The subjects also showed a significant increase of prefrontal $\beta$ activity.

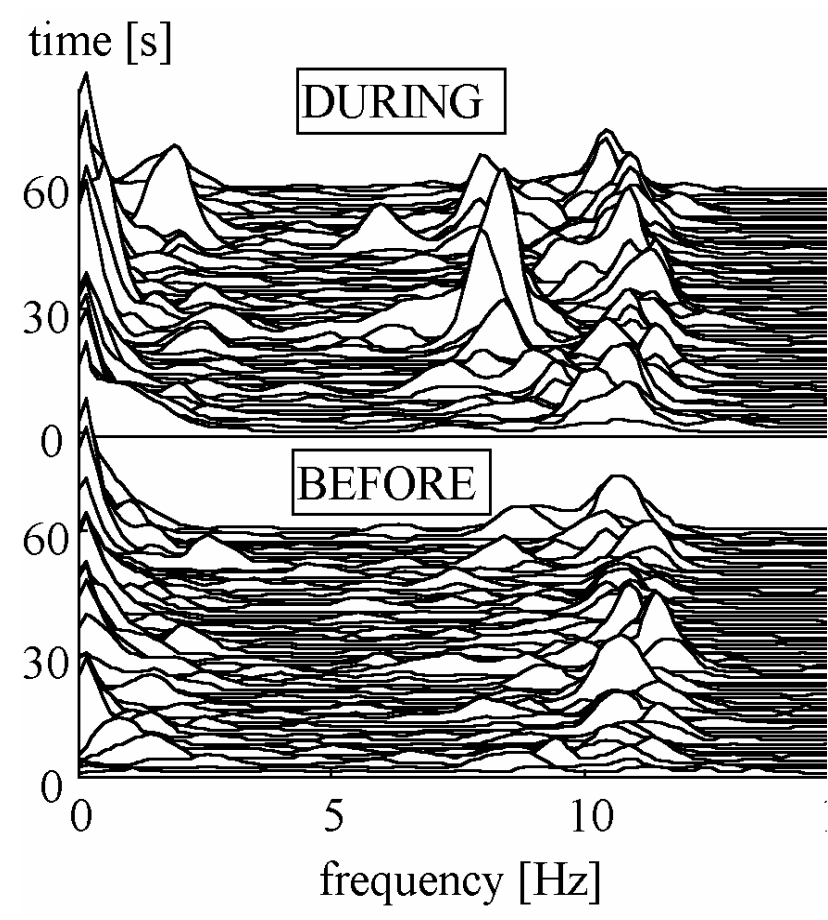

(a)

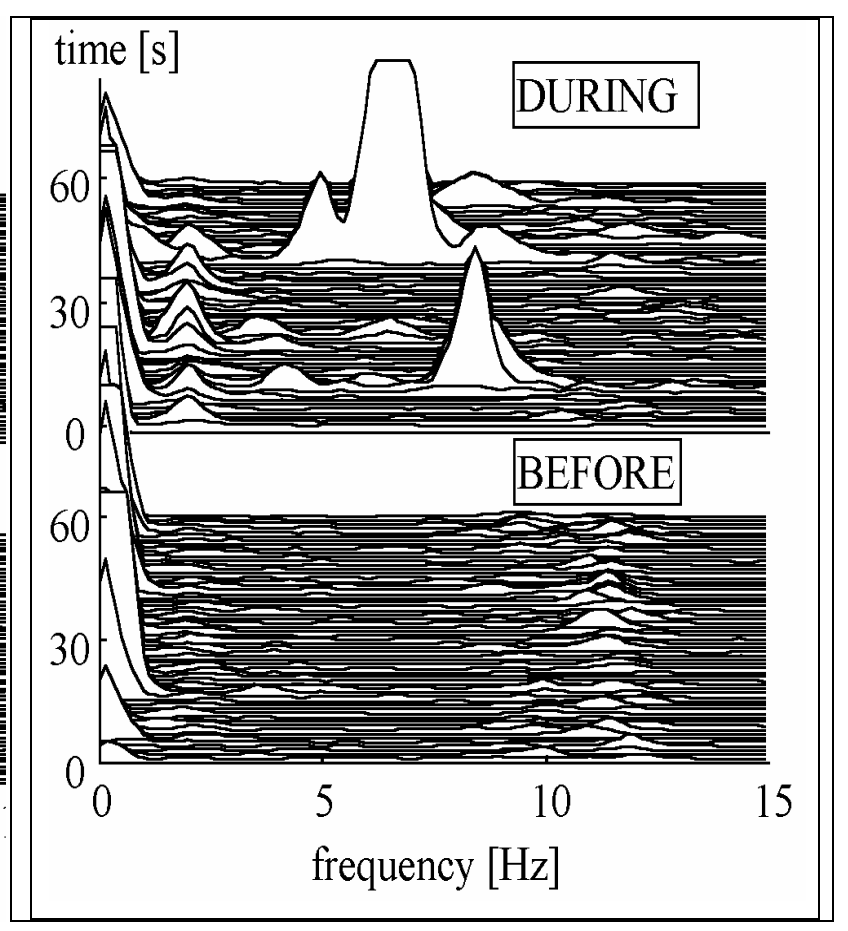

(b)

Figure 4. (a) Compressed power spectral arrays of the EEG for subject 1 obtained from the electrode P3 before and during meditation, showing the slow $\alpha$ waves at $8 \mathrm{~Hz}$; (b) Compressed power spectral arrays of the EEG for subject 12 obtained from the electrode F3 before and during meditation, showing a $\theta$ burst at 6 $\mathrm{Hz}$.
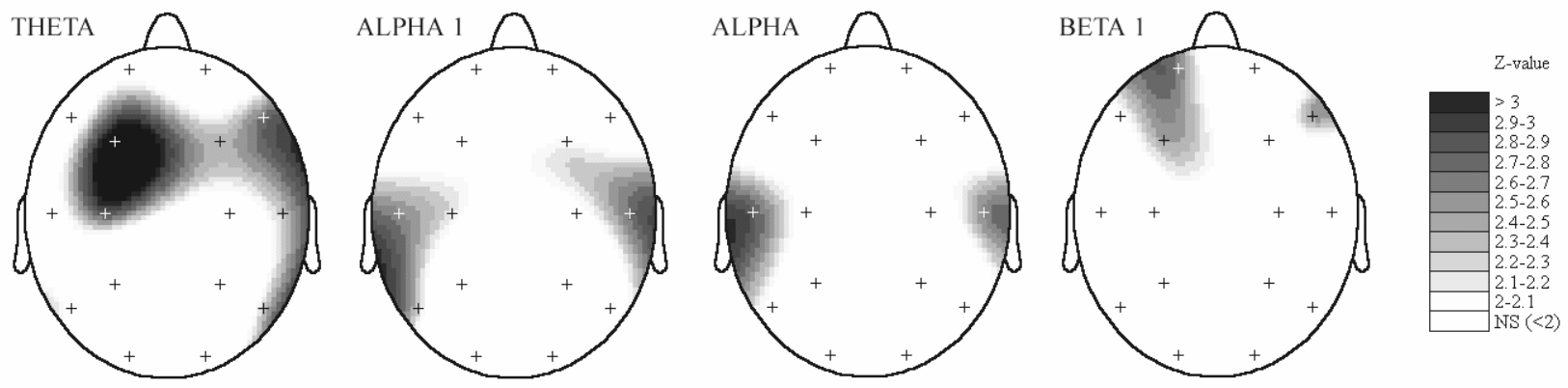

Figure 5. The topographic mapping of significant $(\mathrm{p}<0.05)$ z-values of partial EEG power for each frequency band for the meditation study: $\theta(4-8 \mathrm{~Hz}), \alpha_{1}(8-10.5 \mathrm{~Hz}), \alpha(8-13 \mathrm{~Hz})$, and $\beta_{1}(13-18 \mathrm{~Hz})$; Wilcoxon matched pairs test; inverse distance method. 
Many of the previously published papers have reported physiological changes during meditation that seem to characterize substates of wakefulness [46,52,55-57]. Those changes have been interpreted as a support for the fourth major state of consciousness, the restful alertness state, being a combination of restfulness (increase in $\alpha$ and $\theta$ activity) and alertness (increase in $\beta$ activity). This is in accordance with the Ellias and Grossberg model of neuron [58], which predicts that higher input to brain neural network increases frequency and decreases amplitude of oscillations. In this case, appearance of significant $\theta$ component and the $\alpha$ rhythm slowing may be the result of deprivation of the sensory input. On the other hand, increased $\beta$ power could be a consequence of the increased mental activity.

\section{Musicogenic states}

The study of the perception of music is a paramount example of multidisciplinary research, in which musicians, psychologists, neurobiologists, physicists, and engineers must communicate and work together. This study comprises three broad problem areas [59]: (a) perception of musical tones; (b) interpretation of acoustical information relevant to music; and (c) emotional response to musical messages. In the past two decades, a considerable mutual integration of these three problem areas has taken place, due to the progress in the understanding of general human brain functions, and the recognition that in the conscious state even the simplest perceptual events are bound to trigger operations that involve the brain as a whole.

It should be also pointed out that one of the most profound consequences of the evolution of human brain functions (and human consciousness itself!) has been the emergence of systematic postponing of behavioral goals and rearrangement of behavioral priorities. This led to conflicts between cortical functions and those of the limbic system: while in animals the limbic system is mostly activated by environmental and somatic input, in humans it can also respond to internally evoked images displayed on the cortex during the process of thinking. As motivation and emotion are integral manifestations of limbic function (assuring that all cortical processes are carried out so as to be of maximum benefit of organism, through the extended reticular-thalamic activating system [60]), in humans they can be triggered with no relationship to the instantaneous state of the environment. It is along this line that we should seek a lead toward understanding the human emotional response to music (and to art in general!), when the messages therein seem to be of no obvious survival value [59]. It might even be that deep artistic experiences of spectators could have strong spiritual note through spontaneous spectator's mental addressing on the masterpiece (exciting him in altered state of consciousness), and through it on the illuminating idea related to the artist in the moment of masterpiece creation [9].

This motivated us to address our question on similar possible neurobiological origin of musicogenic altered states of consciousness, induced by deep spiritual music of different cultures [61], and its possible EEG correlates. The analogous more frequently used physical mechanisms for sound-induced altered states of consciousness is an introspective repeating of a certain type of sound or "mantra", which is chosen so as to "resonate" with the structure of an individual nervous system [46]. The sound resonances within the human lobe would be then achieved through a formation of standing sound waves, with principal harmonic (of $\sim 1000 \mathrm{~Hz}$ ) having its maximal amplitude in the centre of the lobe cavity, i.e. around the region of limbic system - therefore inducing the local stimulation of thalamic formation through some mechano-chemical receptors (to be still specified therein).

The study was carried out on 6 healthy adult volunteers. There were one male and five females, whose ages ranged from 18 to 29 years with a mean age of 25 years. All subjects were free of any medication. Prior to the experiment, subjects were informed verbally about all aspects of the experimental procedure.

The four types of spiritual music were provided to the subjects during experiments: (1) The Indian Bhajan in Sanskrit, (2) The Byzantine Easter liturgy in Greek, (3) The Maronit Song in 
Arabian, and (4) Mozart's Requiem in Latin.

The experiment was conducted in a soundproof room, with only one music a day. Each recording session was divided into three sequential periods: (1) relaxing 5 min with eyes closed; (2) listening of the music $10 \mathrm{~min}$; and (3) after listening, $5 \mathrm{~min}$. During those periods three samples, one minute each, were recorded for every subject. The EEG record was stored on a hard disk.

In most cases, during the listening of music, the EEG power decrease is observed in various frequency bands. In the three cases (out of 24), a significant power increase in $\theta$ and $\alpha$ bands is registered, in accordance with an intense aesthetic experience in these cases; the two most prominent spectral arrays before, during, and after the listening of music are shown in Fig. 6 (a, b).

In Table 1, the results of the Wilcoxon matched pairs test for medians of EEG power of all 16 channels, prior and during the listening of music.

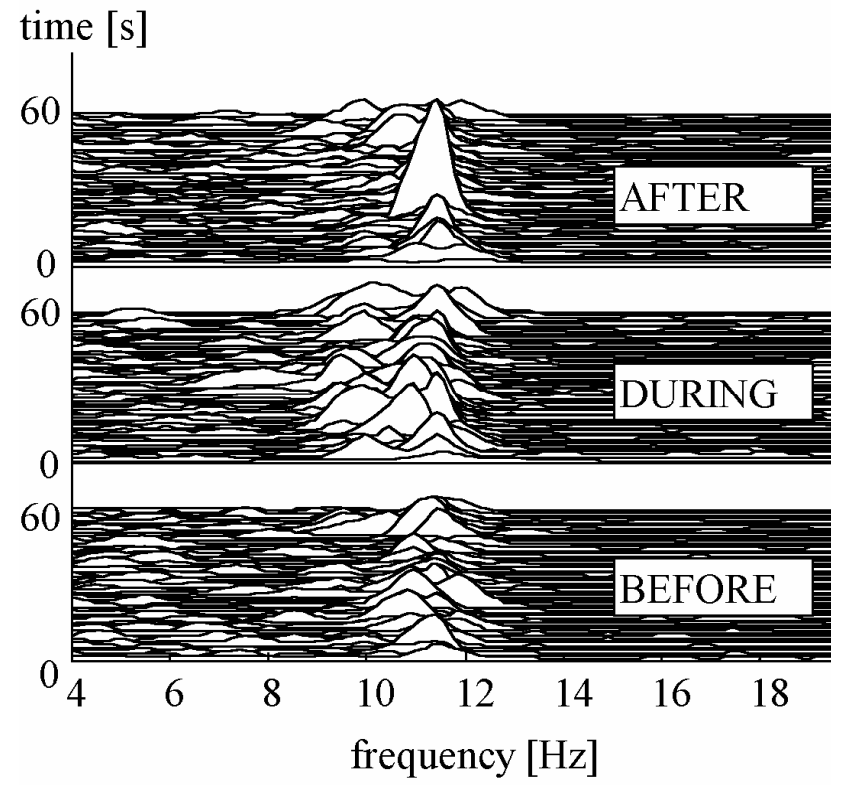

(a)

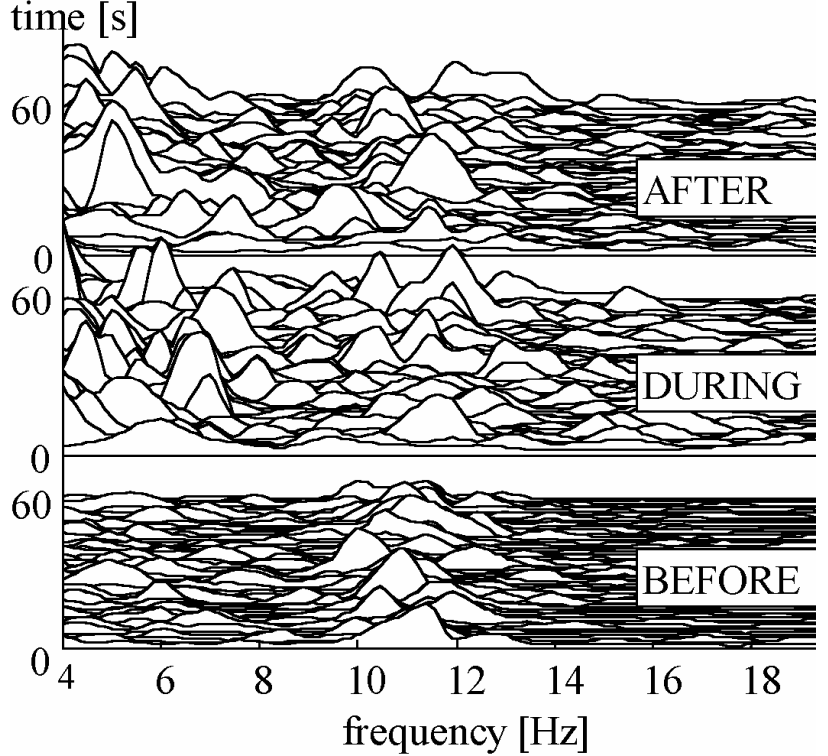

(b)

Figure 6. (a) The spectrogram with the observed EEG power increase in the $\alpha$ band and the appearance of slower $\alpha$ frequencies during the listening of music 1 in channel P3 of subject 3; (b) The spectrogram with the observed high EEG power increase in the $\theta$ band during the listening of music 1 in channel T6 of subject 4 .

Table 1. The EEG power changes during the listening of music.

\begin{tabular}{|c|c|c|c|c|c|c|c|c|c|c|c|c|c|c|c|c|c|c|}
\hline \multirow[b]{3}{*}{ MUSIC } & \multirow{2}{*}{\multicolumn{3}{|c|}{$\frac{\text { SUBJECT } 1}{\text { BAND }}$}} & \multirow{2}{*}{\multicolumn{3}{|c|}{$\frac{\text { SUBJECT } 2}{\text { BAND }}$}} & \multirow{2}{*}{\multicolumn{3}{|c|}{$\frac{\text { SUBJECT } 3}{\text { BAND }}$}} & \multirow{2}{*}{\multicolumn{3}{|c|}{$\begin{array}{c}\text { SUBJECT } 4 \\
\text { BAND } \\
\end{array}$}} & \multirow{2}{*}{\multicolumn{3}{|c|}{$\begin{array}{c}\text { SUBJECT } 5 \\
\text { BAND } \\
\end{array}$}} & \multirow{2}{*}{\multicolumn{3}{|c|}{$\frac{\text { SUBJECT } 6}{\text { BAND }}$}} \\
\hline & & & & & & & & & & & & & & & & & & \\
\hline & $\theta$ & 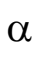 & $\beta_{1}$ & $\theta$ & $\alpha$ & $\beta_{1}$ & $\theta$ & $\alpha$ & $\beta_{1}$ & $\theta$ & $\alpha$ & $\beta_{1}$ & $\theta$ & $\alpha$ & $\beta_{1}$ & $\theta$ & $\alpha$ & $\beta_{1}$ \\
\hline 1 & - & & 0 & 0 & - & 0 & - & + & 0 & + & - & 0 & - & - & - & 0 & - & - \\
\hline 2 & 0 & - & - & - & - & - & - & - & - & 0 & 0 & 0 & - & - & - & $\mathrm{X}$ & $\mathrm{x}$ & $\mathrm{x}$ \\
\hline 3 & $\mathrm{x}$ & $\mathrm{X}$ & $\mathrm{x}$ & + & + & - & - & - & - & 0 & - & 0 & 0 & - & - & - & - & - \\
\hline 4 & 0 & - & - & 0 & - & - & - & - & - & $\mathrm{x}$ & $\mathrm{x}$ & $\mathrm{X}$ & 0 & - & 0 & $\mathrm{x}$ & $\mathrm{x}$ & $\mathrm{x}$ \\
\hline
\end{tabular}

+ sign. increase, - sign. decrease, 0 no sign. changes, $\mathrm{x}$ not recorded 


\section{Healer/healee interaction}

The transitional states of consciousness $[9,40,41,62]$ are presumably the basis of most transpersonal phenomena [18] - being really described by seldom practitioners as not subjected to spatio-temporal limitations $[24,63,64]$ - providing also explanation for their transitional nature and poor reproducibility: they elapse only $\sim 0.1 \mathrm{~s}$, and spontaneous conditions for them are achieved only every 1.5-2 hours, with periodicity of ultradian rhythms which govern the interchange of normal and altered states of consciousness [65]. However, it should be noted that the non-lowdielectric barriers in interaction with the low-dielectric displaced part of ionic acupuncture system are helping in overcoming themselves in such induced transitional states - quite opposite to normal experience in usual mechanical interactions - enabling even their deliberate control and prolongation $[24,63]$ !

It should be also pointed out that the ionic nature of the acupuncture system suggests the possibility that ions in air (prana, qi, pneuma!?) can be physiologically effective [26], just through the acupuncture ionic system and biophysical mechanisms that lie in the basis of acupuncture regulation [1-3] (out of them, the positive ions have an catabolic influence (yang!?) and the negative ones an anabolic influence (yin!?) [26]). So, qi (sometimes erroneously referred as a new kind of biological energy, bioenergy) can be related to ions flowing through the ionic channels of the acupuncture system in the form of MW/ULF ionic currents, with informational content coded in spatio-frequency form of currents and EM fields. It should be pointed out that a lot of experimental phenomena related to external qi gong treatment [23] can be reconciled with the ionic nature of qi. Hence, it seems that the healing process can be related with the transfer of ions between the healer and healee, and/or transfer of the MW/ULF EM information patterns responsible for normal functioning of acupuncture system and overall health $[9,10]$. Also, even distant displacements of healer's ionic structure in remote diagnosis and healing [24] could be expected in transitional states of consciousness.

This was our motivation to start examination [45] of EEG correlates of the healer/healee noncontact interactions, as presumably most intriguing and relatively easily reproducible transpersonal phenomena [23]. This paper presents preliminary results obtained during five healing sessions of one healer. Obtained results will be used to set-up framework of future research.

The study was carried out on one healer and five healthy adult volunteers. There were 3 males and 2 females, whose ages ranged from 24 to 30 years with a mean age of 26 years. All subjects were free of any medication. Prior to the experiment, subjects were informed verbally about all aspects of the experimental procedure.

The experiment was conducted in a soundproof room. Healer and healee were in relaxed state with eyes closed. Subjects had no physical contact. Each recording session was divided into three sequential periods: (1) before the healing session $(2 \mathrm{~min}$ with eyes closed, healer had no activity); (2) during the healing session $3 \mathrm{~min}$; and (3) after the healing session (2 min). During those periods EEGs of healer and healee were recorded and stored on a hard disk

The healer's power spectrum exhibited some changes in $\theta$ region, the greatest being at frontal brain sites (Fig. 7(a)). The healee's power spectrum during the session exibited changes in $\theta$ region at frontal brain sites too (Fig. 7(b)).

The Man-Whitney U-test was used to analyse coherence spectral arrays. Significant changes in 3 out of 5 experiments were observed. Changes were most pronounced at channel T3 (both healer's and healee's). However, due to their small values, medians of coherence time series (30$50 \%$ ) were not taken into consideration. Increase of coherence occurred only in short intervals. Epochs of $4 \mathrm{~s}$, before and during the session were used to estimate maximum coherence value in $\alpha$ and $\theta$ bands.

An example of synchronized EEG signals of healer and healee 3 during the treatment, as well as the corresponding phase diagrams and coherence diagrams are shown in Fig. 8. The phase difference at frequencies with maximum coherence is $180^{\circ}$. 


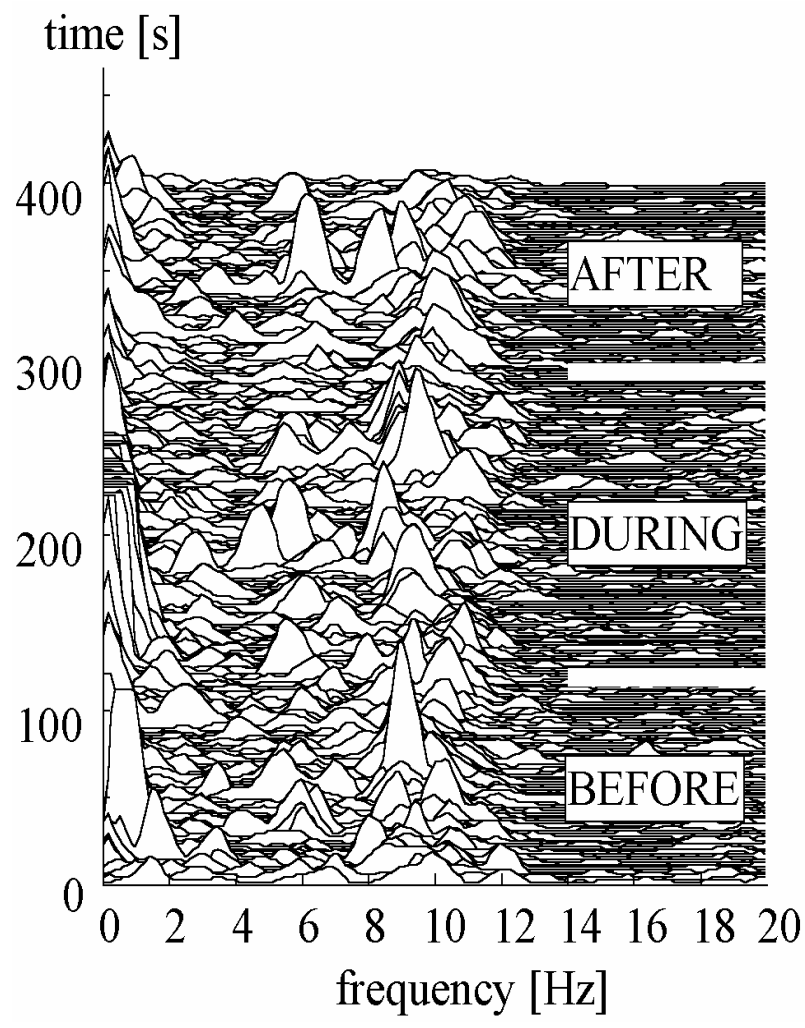

(a)

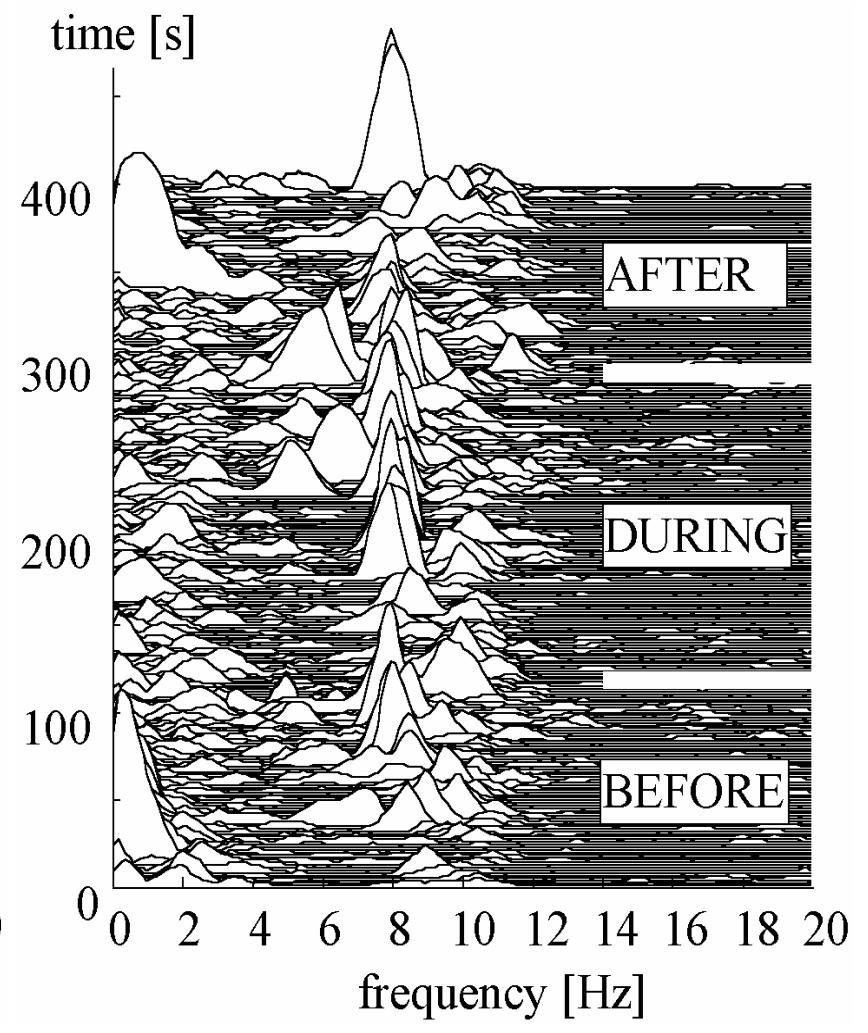

(b)

Figure 7. Spectrogram (channel T3) of (a) healer and (b) healee; increase in power is most pronounced in $\theta$ band during and after the healing session.

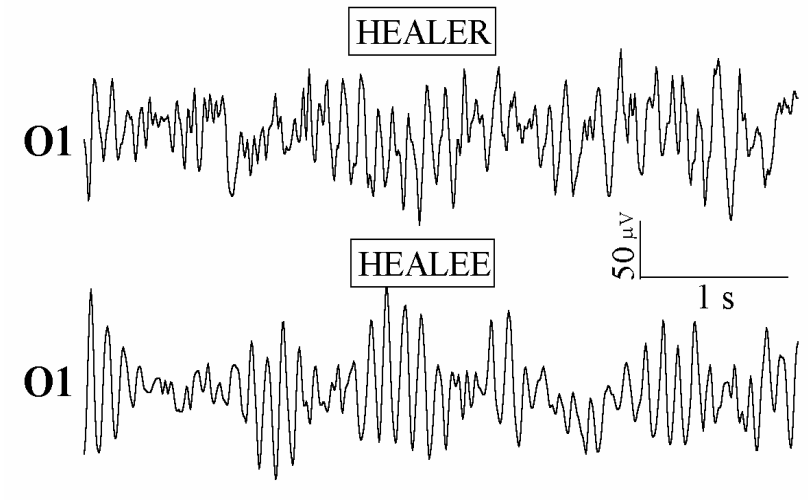

(a)
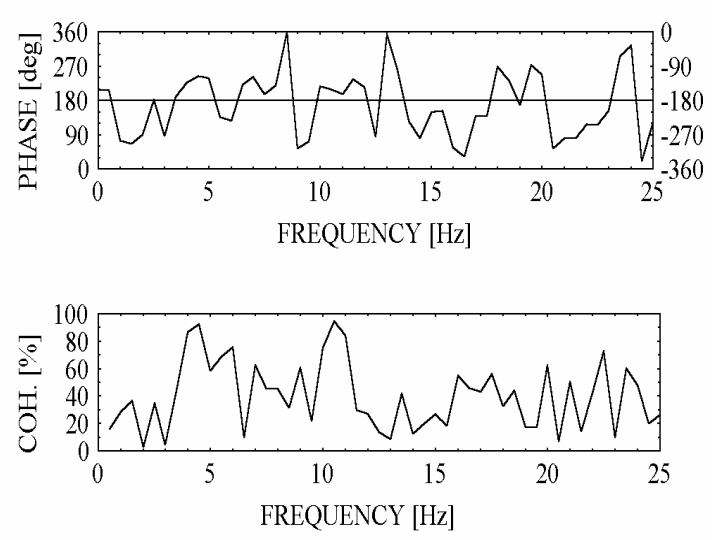

(b)

Figure 8. An example of short-time EEG synchronization with max. coherence; channel O1 of healer and subject 3; plots of (a) original signal and (b) phase and coherence diagrams. 


\section{Alertness/Drowsiness}

Two interesting and to great extent related problems are automatic scoring of sleep stages, and detection of EEG segments of reduced vigilance level during awake stages.

The same standards for the visual classifying of sleep stages have been in use over 30 years $[66,67]$. These standards suffer from severe limitations, and therefore automatic procedures that implement them cannot give satisfying results. Hence, despite the advance of computer technology over the past decades, automatic scoring of sleep stages is far from being solved till now [68]. Man/machine agreement of even the best methods is limited by poor interscorer agreement obtained by comparing results of visual inspection of different electroencephalographers.

The situation is even more vague with the detection of reduced vigilance level segments from EEG recordings. Different approaches have been used to classify the degree of alertness during the awake stages, from subjective ratings, performance tests and neurophysiological measures. Subjective ratings are unreliable because of the fact that feelings such as alertness or drowsiness are poorly defined. Methods based on monitoring performance level like measuring reaction time to some stimuli, cannot give satisfying results as such measurement itself influences the subject's vigilance. It is evident, therefore, that it is the EEG on which the vigilance level estimation should be based. However, there are no universally accepted standards for visual classification of vigilance level during the awake stages, based on EEG traces. This is in contrast to the fact that the changes in EEG frequency bands, and occurrence of patterns assigned to changes of vigilance level, are assumed to be known. Different authors have used different approaches to combine these known characteristic changes in order to form meaningful rules, so further efforts should be obviously directed toward modeling underlying biological processes [68].

An effort in this work is done toward finding underlying functional relationship between power spectrum fluctuations related to changes of vigilance level (not used predetermined relationships) in order to estimate vigilance level. This is done by means of neural network classifier. Employing neural network classifier as a structure with modifiable parameters is of benefit for the following reasons: (a) underlying relationships which are assumed to exist, are not known, and are to be found; (b) by supplying the neural network with training sets obtained from recordings on single subjects, the network "learns" individual patterns characteristic for lower vigilance; and (c) the method can be adjusted to correspond to the results obtained by visual inspection of different experts. This allows interaction between electroencephalographers and machine, that will lead to better understanding of underlying principles and therefore to more efficient standards.

Electroencephalograms of 30 healthy young subjects were recorded. Subjects were aged 2028 (median: 25), 22 males and 8 females, and have passed neurological screening. Uniformly aged subjects were chosen because EEG changes with age, and universal rules for automatic detection of vigilance level should require much bigger experimental group. Recordings were performed between 2-4 p.m. Subjects were not sleep deprived, nor had any deviations from their usual circadian cycle, and they took no drugs.

Recordings lasted from 15 to 30 minutes, depending on subject's level of drowsiness i. e. frequency of occurrence of low vigilance segments on corresponding EEG traces. Subjects were not allowed to fall asleep (i. e. further from stage I of slow wave sleep - drowsiness). We required at least two minutes of low vigilance level in total EEG time. EEG signals were analyzed off-line, and epochs without artifacts, characteristic for full wakefulness and for lower vigilance were cut by experienced electroencephalographer and pasted to form two-minute long segments used, one as a representative of normal fully awake state (alert wakefulness), and another one as a representative of drowsy wakefulness.

Single layer perception neural network was used for vigilance level assessment. Training and test input vectors were made from one two-minute long segment of alert wakefulness and one of drowsy wakefulness. The state of alert wakefulness was assigned the value of 1 , and the state of 
drowsy wakefulness was assigned the value of 0 . These values were supplied to the network as desired output values during the training session.

Segments of $30 \mathrm{~s}$ of both recording were used to construct training sets. The network was tested on the rest $90 \mathrm{~s}$ of each recording. To achieve high processing speed it was necessary to make input vectors of low dimensionality. Also, it was necessary to make the components of input vectors to be easy to compute. Thus, the power spectrum characteristics were used to form input vectors. Spectral analysis is the most important and most common technique in EEG time series analysis [69]. Due to great inter-individual variations in total power, the relative values were computed, i. e. the power in each frequency band was divided by the total power in all bands.

The slowing of dominant $\alpha$ frequency and widening of $\alpha$ peak are assumed to be the most important signs of drowsiness. However, it is not possible to see these events through changes of relative power in $\alpha$ band. Therefore, $\alpha$ band was divided into two bands, $\alpha_{1}(7.5-9.5 \mathrm{~Hz})$ and $\alpha_{2}$ $(9.75-12.5 \mathrm{~Hz})$. These boundaries are carefully chosen so that values of relative power in these bands carry the same amount of information, as does the shift of the dominant $\alpha$ frequency, and widening of $\alpha$ peak.

Boundaries of frequency bands used were as follows: $0.5-3.25 \mathrm{~Hz}(\delta), 3.5-7.25(\theta), 7.5-9.5$ $\mathrm{Hz}\left(\alpha_{1}\right), 9.75-12.5 \mathrm{~Hz}\left(\alpha_{2}\right), 12.75-18 \mathrm{~Hz}\left(\beta_{1}\right)$, and $18.25-25 \mathrm{~Hz}\left(\beta_{2}\right)$.

Since it is shown in earlier studies [70] that significant differences in EEG patterns between hemispheres during the drowsiness do not occur, power spectrum was computed from the one hemisphere (right).

Slight vigilance fluctuations can occur in time periods as short as few seconds. Therefore, short time epochs were used to compute power spectrum characteristics. The use of 4-s long epochs provided a good compromise between time and frequency resolution. Frequency resolution was $0.25 \mathrm{~Hz}$, which was good enough to successfully divide spectrum into bands. In order to improve time resolution, epoch overlapping of $2 \mathrm{~s}$ was used. Thus, 28 epochs were used for training, and 84 epochs were used for testing.

In order to find the power spectrum characteristics that best reflect the expert's knowledge used for visual classification results from previous studies were consulted as well [70-76]. Characteristics that in combination with perceptron neural net proved to give the most satisfactory results are shown in Table 2. These values were used to form input vectors for both training and testing. Input vectors during the training phase were supplied to the network along with the desired values of output. During training cycle, perceptron neural network assigned the weighting values to each component of the input vectors. These weights were used in testing phase, in order to estimate the level of vigilance. Training network on each subject enabled the network to "learn" individual fluctuations in EEG spectrum.

Table 2. Combinations of power spectrum values and electrode positions used to form input vectors.

\begin{tabular}{lccccccccccc}
\hline \hline $\begin{array}{l}\text { FREQUENCY } \\
\text { BAND }\end{array}$ & $\alpha_{2}$ & $\alpha_{1}$ & $\theta$ & $\theta$ & $\beta_{1}$ & $\beta_{1}$ & $\beta_{1}$ & $\beta_{2}$ & $\beta_{2}$ & $\beta_{2}$ & $\theta / \alpha$ \\
\hline $\begin{array}{l}\text { ELECTRODE } \\
\text { POSITION }\end{array}$ & $\mathrm{O} 2$ & $\mathrm{O} 2$ & $\mathrm{O} 2$ & $\mathrm{~F} 4$ & $\mathrm{~F} 4$ & $\mathrm{C} 4$ & $\mathrm{O} 2$ & $\mathrm{~F} 4$ & $\mathrm{C} 4$ & $\mathrm{~T} 6$ & $\mathrm{O} 2+\mathrm{F} 4+\mathrm{F} 8+\mathrm{C} 4+\mathrm{T} 4$ \\
\hline
\end{tabular}

Numerical results were computed on a standard PC. Procedures for data manipulation and neural network implementation were developed in Matlab 4.2 environment [77]. Training and testing of the network took a few seconds of CPU time on PC 486/100 MHz.

Results of the procedure applied for five typical subjects are summarized in Table 3. 
Table 3. Results of perceptron classification of vigilance level, for five typical subjects.

\begin{tabular}{cccccccc}
\hline \hline & \multicolumn{3}{c}{ TRAINING PHASE } & & \multicolumn{3}{c}{ TESTING PHASE } \\
\cline { 2 - 3 } SUBJ. & $\mathrm{N}^{\mathrm{O}}$ OF & $\mathrm{N}^{\mathrm{O}}$ OF VECTORS & ERROR & & $\mathrm{N}^{\mathrm{O}}$ OF & $\mathrm{N}^{\mathrm{O}}$ OF VECTORS & ERROR \\
& VECTORS & SUCC. LEARNED & RATE $(\%)$ & VECTORS & SUCC. CLASSIFIED & RATE (\%) \\
\hline 1 & 28 & 28 & 0 & 84 & 79 & 5.95 \\
2 & 28 & 28 & 0 & 84 & 81 & 3.57 \\
3 & 28 & 28 & 0 & 84 & 83 & 1.19 \\
4 & 28 & 28 & 0 & & 84 & 81 & 3.57 \\
5 & 28 & 28 & 0 & 84 & 84 & 0.00 \\
\hline
\end{tabular}

\section{CONCLUSION}

Since conventional partial methods have failed to prevent and treat numerous modern psychosomatic diseases, having its convenient ground in modern men exposed to everyday stress, new approaches are required oriented in healing a man as a whole (not a disease which is only a symptom of the disordered wholeness), which will include holistic traditional methods, focused on human acupuncture system and consciousness as significant psychosomatic global regulators of health, but supported with contemporary biomedical technologies. One of them is Microwave Resonance Therapy (MRT), which represents a synthesis of the ancient Chinese traditional knowledge in medicine (acupuncture) and recent breakthroughs in biophysics.

Affecting the appropriate acupuncture points by the generation of high frequency microwaves $(52-78 \mathrm{GHz})$, remarkable clinical results of the treatment are being achieved in over 60 pathologies during the last decade. In this paper the biophysical basis of the MRT were pointed out: the quantum-like macroscopic coherent characteristics of the MRT (sharply-resonant sensory response of the disordered organism, extremely low-intensity and low-energy non-thermal biologically efficient MW radiation, and negligible MW energy losses down acupuncture meridians) suggest that healthy condition might be considered as an absolute minimum (ground state) of the nonlocal selfconsistent macroscopic quantum potential of the organism, some disorders of an acupuncture system corresponding to higher minimums of the (spatio-temporally changeable) potential hypersurface in energy-configuration space, which possibly explains the higher sensory responses of the more excited (more disordered) acupuncture system, and poor MRT sensory response of the healthy acupuncture system being already in the ground state. Such a picture also supports the EM/ionic "optical" ultralowfrequency modulated MW quantum holographic neural network-like function of the acupuncture system (similar to complex-valued oscillatory holographic Hopfield-like neural networks), and its essential relation to consciousness, as strongly suggested from biophysical modeling of altered states of consciousness. Finally, our electroencephalographic investigations showed more or less characteristic EEG features in 28 subjects of relaxation induced by microwave resonance therapy applied to corresponding acupuncture points (with slightly decreased EEG power in all frequency bands especially in the left central region, which can be ascribed to higher activation of the stimulated left circulatory part of the acupuncture system; it should be also noted that persons not previously subjected to this treatment responded stronger, presumably as a consequence of the more dysbalanced acupuncture system).

Regarding some other activities which may alter consciousness artificially or spontaneously, we obtained more or less characteristics EEG changes in 25 subjects of transcendental meditation (increased beta power in prefrontal region, increased theta power in left frontal and right temporal regions, and increased alpha power in both temporal regions), 6 subjects with 4 types of spiritual music provided to induce musicogenic states (with significant changes in only 3 cases out of 24 , where increased theta and alpha power was observed in only those subjects 
who have described their musical experiences as very pleasant), 5 healer/healee noncontact interactions (with increase in the maximum mean coherence of their EEG patterns in the alpha band observed only in short $4 \mathrm{~s}$ time intervals), and 30 subjects for monitoring alertness/drowsiness level (with implemented automatic procedure of the neural network classifier to assess the correlation between EEG power spectrum fluctuations related to changes of vigilance level, demonstrating linear separability of the states of alert wakefulness and drowsy wakefulness, allowing very fast data processing and possible real time applications in clinical practice). This demonstrates that EEG may permit rapid and reproducible identification of different states of consciousness, useful in evaluating the effectiveness of consciousness altering techniques and related stress reduction.

\section{REFERENCES}

1. Omura, Y.: Acupuncture Medicine, Japan Publ. Inc., Tokyo, 1982; Chinese Acupuncture and Moxibustion, C. Xinnong, ed., Foreign Languages Press, Beijing, 1987.

2. Portnov, F.G.: Electropuncture Reflexotherapeutics, Zinatne, Riga, 1982, in Russian.

3. Škokljev, A.I.: Acupuncturology, ICS, Belgrade, 1976, in Serbian; Škokljev, A.I.: Physiological mechanisms of the acupuncture, in Exogenous and Endogenous Biological Interactions: Biophysical and Biochemical Aspects, D. Raković and Dj. Koruga, eds., Molecular Machines Research Center \& IHIS FST Center, Belgrade, 1998, in Serbian; Opačić, M., Batinić, J., Janković, S., Škokljev, A., Raković, D., Kozić, Dj. and Koruga, Dj.: Neural network and YinYang modeling of normal and cancer cell functioning, Archive of Oncology 5: 129-134, 1997.

4. Johari, H.: Breath, Mind, and Consciousness, Destiny Books, Rochester, Vermont, 1989.

5. Kandel, E.R.,. Siegelbaum, S.A and. Schwartz J.H.: Synaptic transmission, in Principles of Neural Science, E.R. Kandel, J.H. Schwartz and T.M. Jessell, eds., Elsevier, New York, 1991, Ch. 9.

6. Mashansky, V.F., Markov, Yu.V., Shpunt, V.H., Li, S.E. and Mirkin, A.S.: Topography of gap junctions in human skin and its possible role in non-nervous transition of information, Archive of Anatomy, Histology, and Embriology 84 (3): 53-60, 1983, in Russian; Li, S.E., Mashansky, V.F. and Mirkin, A.S.: Lowfrequency wave and vibrational processes in biosystems, in Vibrational Biomechanics. Using of Vibrations in Biology and Medicine, Part I: Theoretical Bases of Vibrational Biomechanics, K.V. Frolov, ed., Nauka, Moscow, 1989, Ch.3, in Russian.

7. Đorđević, D.: Electrophysiological Investigations of the Mechanisms of Reflexotherapeutics, M.Sc. Thesis, Medical Faculty of Belgrade University, 1995, Ch. 1.2, in Serbian; Đorđević, D.: Epithelial conception of genesis of the system of vital meridians and their acupuncture points Serbian Journal of Acupuncture 1: 17-22, 1998, in Serbian.

8. Benett, M.V.L., Barrio, L.C., Bargiello, T.A., Spray, D.C.,E. Hertzberg and Saez, J.C.: Gap junctions: New tools, new answers, new questions, Neuron 6: 305-320, 1991.

9. Raković, D.: Neural networks, brainwaves, and ionic structures: Acupuncture vs. altered states of consciousness, Acupuncture \& Electro-Therapeutics Research, The International Journal 16: 88-99, 1991; Raković, D.: Fundamentals of Biophysics, Grosknjiga, Belgrade, 1994, 1995, Chs. 5-6, in Serbian; Raković, D.: Brainwaves, neural networks, and ionic structures: Biophysical model for altered states of consciousness, in Consciousness: Scientific Challenge of the $21^{\text {st }}$ Century, D. Raković and Dj. Koruga, eds., ECPD, Belgrade, 1995, 1996; Raković, D.: Hierarchical neural networks and brainwaves: Towards a theory of consciousness, in Proceedings of ECPD Workshop Brain \& Consciousness, Lj. Rakić, G. Kostopoulos, D. Raković and Dj. Koruga, eds., Belgrade, 1997, and references therein.

10. Raković, D.: Biophysical basis of traditional medicine and traditional psychology, Serbian Journal of Acupuncture 1: 6-12, 1998, in Serbian; Raković, D.: Traditional medicine and traditional psychology: Biophysical bases of psychosomatic disorders and transpersonal stress reprogramming, in Theory of Functional Systems - Basic and Clinical Approach, Proceedings of $4^{\text {th }}$ Yugoslav-Russian Conference, B. Lažetić, ed., Palić, 1998; Raković, D. and Jovanović- 
Ignjatić, Z.: Microwave resonance therapy and acupuncture: New prospects for traditional medicine, $14^{\text {th }}$ Ann. Int. Symp. Acup. \& Elec. Therap., New York, 1998; Jovanović-Ignjatić, Z. and Raković, D.: A review of current research in microwave resonance therapy: Novel opportunities in medical treatment, Acup. \& Electro-Therap. Res., The Int. J., 1998, submitted.

11. Adey, W.R.: Frequency and power windowing in tissue interactions with weak electromagnetic fields, Proceedings of the IEEE 68: 119-125, 1981, and references therein.

12. Pomeranz, B.: Acupuncture research related to pain, drug addiction and nerve regeneration, in Scientific Bases of Acupuncture, B. Pomeranz and G. Stux, eds., Springer, Berlin, 35-52, 1989.

13. Fischer, G.: Grundlagen der Quanten-Therapie, Hecataeus Verlagsanstalt, Triesenberg, 1996.

14. Sit'ko S.P. and Mkrtchian, L.N.: Introduction to Quantum Medicine, Pattern, Kiev, 1994.

15. In Biological Aspects of Low Intensity Millimeter Waves, N.D. Devyatkov and O.V. Betskii, eds., Seven Plus, Moscow, 1994.

16. The key problem of any future theory of consciousness is how to incorporate altered states of consciousness (REM sleep, meditation, hypnosis, psychedelic drug influence, some psychopathological states, near-death experiences, ...) within a new paradigm. It should be pointed out that purely biochemical mechanisms of the extended reticular-thalamic activating system (serving as a selector and amplifier of the conscious content out of many other currently processed nonamplified contextual unconscious contents) are not accelerated up to several orders of magnitude - as the subjective time sense is dilated in altered states of consciousness in respect to the normal awake state. According to the biophysical model for altered states of consciousness $[9,10]$, the EM component of ULF brainwaves, related to "subjective" reference frame of consciousness, enables perfect fitting with narrowed-down limits of conscious capacity in normal awake states and very extended limits in altered states of consciousness - due to the biophysical relativistic mechanism of dilated subjective time base. In this model, consciousness is subtle internal display in the form of EM component of ULF brainwave ionic currents, in which a complete information (both conscious and unconscious) is permanently coded from brain's neural networks, as a spatio-temporal pattern resulting from changes of the electrosynaptic interconnections in the neural networks of the brain. Then, according to this model, altered states of consciousness are a consequence of partial displacement of the ionic acupuncture system outside the body (when the embedded EM component of ULF brainwaves is propagating through this weakly ionized structured gaseous medium of low-dielectric relative permittivity, $\varepsilon_{r} \approx 1$ ), while normal states of consciousness (alert state, non-REM sleep,...) are achieved when there are no such displacements (when brainwaves are propagating only through the structured brain tissue of high-dielectric relative permittivity, $\varepsilon_{r} \gg 1$ ). The displaceable (from the body) acupuncture ionic structure in this model must have a form of "optical" MW neural network, for continually inflowing ULF brainwave information from the brain's neural networks to be "subjectively" recognized, through ULF modulation of the MW neural network. Also, in altered states of consciousness, the displaced weakly ionized gaseous "optical" MW neural network behaves also as an visual sensor (through visual optical modulation of the MW neural network), which can even perceive an environment extrasensory, as actually reported by reanimated persons. Even most peculiar spatio-temporal transpersonal interactions are predicted in transitional states of interchange of normal and altered states of consciousness (when brainwaves traverse from high-dielectric $\left(\varepsilon_{r}>1\right)$ to low-dielectric $\left(\varepsilon_{r} \approx 1\right)$ state or vice versa, the relative velocity $v=c_{0} / \sqrt{\varepsilon_{r}}$ of "subjective" reference frame being therefore subjected to abrupt change in short transitional period $\tau \sim 0.1 \mathrm{~s}$, with "subjective frame" acceleration $\sim c_{0} / \tau$ $\sim 10^{9} \mathrm{~m} / \mathrm{s}^{2}$ ) - due to the relativistic generation of so-called wormholes in highly noninertial "subjective" reference frame - fully equivalent, according to Einstein's Principle of equivalence, to extremely strong gravitational fields where generation of wormholes (or Einstein-Rosen space-time bridges, whose entrance and exit might be in very distant space-time points) is theoretically predicted [17]. It should be pointed out that apart from the EM field, the displaced part of ionic acupuncture system (in the form of ionic neural network, having the "optical" 
sensory function), must also be tunneled in such (acausal) interactions of consciousness with distant events in space-time. These transitional states of consciousness are presumably also the basis of most transpersonal phenomena [18] - being actually described by rare practitioners as not subjected to spatio-temporal limitations - providing also an explanation for their transitional nature and poor reproducibility: they elapse in $\sim 0.1 \mathrm{~s}$, and spontaneous conditions for them are achieved only every $1.5-2$ hours, with periodicity of ultradian rhythms which govern the interchange of normal and altered states of consciousness.

17. Thorne, K.S.: Black Holes and Time Warps: Einstein's Outrageous Legacy, Picador, London, 1994, Ch. 14, and references therein.

18. Jahn, R.G.: The persistent paradox of psychic phenomena: An engineering perspective Proc. IEEE 70: 136-170, 1982.

19. Nuccitelli, R.: The involvement of transcellular ion currents and electrical fields in pattern formation, in Pattern Formation, G.M. Malacinski, ed., Macmillan, New York, 1984.

20. McCaig, C.D.: Spinal neurite regeneration and regrowth in vitro depend on the polarity of an applied electric field, Development 100: 31-41, 1987.

21. Shang, C.: Singular point, organizing center and acupuncture point, American Journal of Chinese Medicine 17: 119-127, 1989.

22. Kenyon, J.N.: Modern Techniques of Acupuncture, Thorsons, Wellingborough, 1983.

23. Omura, Y., Lin, T.L., Debreceni, L., Losco, B.M., Freed, S., Muteki, T. and Lin, C.H.: Unique changes found on the qi gong (chi gong) master's and patient's body during qi gong treatment: Their relationships to certain meridians \& acupuncture points and the re-creation of therapeutic qi gong states by children \& adults, Acupuncture \& Electro-Therapeutics Research, The International Journal 14: 61-89, 1989.

24. Markides, K.C.: Fire in the Heart. Healers, Sages and Mystics, Paragon House, New York, 1990.

25. Vasilyev, L.L.: Theory and Practice of Aeroionic Therapy, Leningrad, 1951, in Russian; Vasilyev, L.L.: Influence of Aeroions on Organism, Leningrad, 1960, in Russian; Minkh, A.A.: Air Ionization and Its Hygienic Significance, Medgiz, Moscow, 1963, in Russian; Shilkin, A.A., Gubernskii, Yu.D. and Mironov, A.M.: Aeroinic Regime in Buildings, Stroyizdat, Moscow, 1988, in Russian.

26. Krueger, A.P.: Preliminary consideration of the biological significance of air ions, Scientia 104: $1-17,1969$.

27. Physiological significance of the chemical nature of negative and positive aeroions is still not clear. Russian investigations showed [25] that functional effect of ions of the same polarity but different chemical composition (small gaseous anions $\mathrm{O}_{2}^{-}, \mathrm{O}_{3}{ }^{-}, \mathrm{H}_{2} \mathrm{O}^{-}, \ldots$ and cations $\mathrm{NO}^{+}$, $\mathrm{H}_{2} \mathrm{O}^{+}, \mathrm{CO}_{2}^{+}$, ..., heavy aqueous complexes of anions $\mathrm{O}_{2}{ }^{-}\left(\mathrm{H}_{2} \mathrm{O}\right)_{\mathrm{n}}, \ldots$ and cations $\mathrm{H}_{3} \mathrm{O}^{+}\left(\mathrm{H}_{2} \mathrm{O}\right)_{n}$, ..., etc.) is basically equal, implying that aeroinic action is achieved by yielding its charge to biological object. However, it was also shown [28] that negative ions have physiological influence on the increase of tracheal ciliary rate in rabbits only in presence of $\mathrm{O}_{2}$ in air, while positive ions have physiological influence on the decrease of tracheal ciliary rate in rabbits only in presence of $\mathrm{CO}_{2}$ in air - implying possibly physiological significance of $\mathrm{O}_{2}^{-}$and $\mathrm{CO}_{2}{ }^{+}$ aeroions in nostrils, as necessary precursors for dominating inhaling of negative aeroions by ida and positive aeroions by pingala (in their alternating $\sim 1$ hour active phases, respectively), with their corresponding terminations in left and right nostrils, in swara yoga physiology [4]. So, in nasal ida-phase (known also as Shakti, feminine, lunar, or alkaline phase [4], with left nostril activated, and whole left half of the body and right brain hemisphere too), in exhaling through (anabolic) ida in its nasal vicinity should dominate gaseous phase with less exhaled $\mathrm{CO}_{2}{ }^{+}$and therefore relatively more gaseous $\mathrm{O}_{2}^{-}$therein, which additionally stimulates dominant absorption of negative aeroions by active left nostril, supporting ida-phase backward; on the other hand, in nasal pingala-phase (known also as Shiva, masculine, solar, or acidic phase [4]), with right nostril activated, and whole right half of the body and left brain hemisphere too), in exhaling through (catabolic) pingala in its nasal vicinity should dominate gaseous phase with 
more exhaled $\mathrm{CO}_{2}^{+}$and therefore relatively less gaseous $\mathrm{O}_{2}^{-}$therein, which additionally stimulates dominant absorption of positive aeroions by active right nostril, supporting pingalaphase backward. This picture is in accordance with the viewpoint of successive dominant flow of (negative) anions through the left circulatory part of the acupuncture system, and (positive) cations through the right circulatory part of the acupuncture system $[9,10]$, with normal ultradian periodicity $\sim 2$ hours (i.e. $\sim 1$-hour dominance for left and right phases, alternately: ida-pingala-...[4]).

Although this rhythm is not apparently in phase with $\sim 24$-hour acupuncture rhythm of successive dominance of 12 paired meridians (i.e. 2-hour dominance for each organ-related meridian with corresponding yin or yang functions in the following order: yin-yin-yang-yang...[1-3]), it might be that every $\sim 2$-hour organ-related acupuncture phase (either yin or yang) needs complete $\sim 2$-hour nasal phase (ida-pingala) in order to balance activities of the corresponding organ-related pair of symmetrical left and right meridians, to enable both regenerative (anabolic, ida-like left meridian) and degradative (catabolic, pingala-like right meridian) organ functions, contributing finally to either yin or yang overall corresponding organ-effect from the viewpoint of Chinese traditional medicine [1-3]. This ionic interpretation of acupuncture system can be tested, in principle, by subtle electric and magnetic differences between the left and right symmetrical meridians of the acupuncture system, and modulation in their electrical activities with ultradian rhythm periodicity, as stated above.

The biophysical ionic interpretation of the acupuncture system can account for the origin of Chinese therapeutic yin-yang/tonification-sedation rules through the type/rotation of the needles: yin syndrome (excess of negative ions in some left meridian) is tonified by taking in positive ions from air or taking off negative ions from the meridian (through Ag needles working as anode) i.e. by rotating needles in counterclockwise direction on the left meridian (thus closing its gap junction channels for flow of negative ions) or clockwise direction on the corresponding right meridian (thus openning its gap junction channels for flow of positive ions); and yang syndrome (excess of positive ions in some right meridian) is sedated by taking in negative ions from air or taking off positive ions from the meridian (through Au needles working as cathode) i.e. by rotating needles in counterclockwise direction on the right meridian (thus closing its gap junction channels for flow of positive ions) or clockwise direction on the corresponding left meridian (thus openning its gap junction channels for flow of negative ions).

On the other hand, some other Chinese therapeutic rules need taking into account functional physiological interactions between acupuncture system and nervous and humoral systems. For instance, Chinese therapeutic husband-wife rule (yang-yin/left-right) [1] is apparently opposite to Indian functional rule (Shakti-Shiva/left-right) [4] or our biophysical ionic rule (negativepositive/left-right), presumably as a consequence of the acupuncture therapeutic action on the one side of the body to produce effect on the other side by affecting the phase of (brain and nasal) ultradian rhythm, followed by subsequent intermediate contralateral brain-body neurophysiological effect. Regarding some other Chinese therapeutic rules (five elements, mother-child/regulation cicle/Sheng, destruction cicle/Ko, middle day-middle night, centrifugalcentripetal meridians etc.) [1], they are presumably based on intermediate humoral physiological effects between spatio-temporally nonsubsequent meridian-related organs.

Finally, from the functional viewpoint $[1,4]$, it seems that Chinese ren and du central meridians correspond to Indian ida and pingala nadis, respectively, which start and intersect upward (with shushumna nadi in Indian tradition too, having not its Chinese counterpart meridian!?) in first six chakras, save for the seventh highest chakra [4]. Also, 14 Chinese meridians (12 paired and 2 central) might be functionally related to 7 Indian chakras: (1) ren meridian vs. muladhara chakra; (2) kidney/bladder meridians pair vs. svadhishthana chakra; (3) spleen/stomach maridians pair vs. manipura chakra; (4) hearth/small intestine \& pericardium/triple energizer meridians pairs vs. anahata chakra; (5) lung/large intestine meridians pair vs. vishuddha chakra; (6) liver/gallbladder meridians pair vs. ajna chakra; and (7) du meridian vs. sahasrara chakra. 
28. Krueger A.P. and Smith, R.F.: Effects of gaseous ions on tracheal ciliary rate, Proceedings of Society for Experimental Biology 98: 412-414, 1958.

29. Zalyubovskaya, N.P.: An Estimation of Effects of Millimeter and Submillimeter Microwawaves upon Various Biological Objects, M.Sc. Thesis in Biological Sciences, Kharkov State University, 1970, in Russian.

30. Devyatkov, N.D.: Influence of the millimeter wavelength range electromagnetic radiation upon biological objects, Soviet Physics - Uspekhi 110: 452-454, 1973, also, see pp. 455-469 in this volume.

31. Andreyev, Ye.A., Bely, M.U. and Sit'ko, S.P.: Manifestation of characteristic eigenfrequencies of human organism, Application for the Discovery to the Committee of Inventions and Discovery at the Council of Ministers of the USSR, No. 32-OT-10609, 22 May 1982, in Russian.

32. Cherkasov I.S. and Nedzveckii, S.V.: A therapy of disordered biological tissues, Authors Application No. 733697, USSR, 1980.

33. In Miscellany of Methodological Recommendations and Regulations in Microwave Resonance Therapy (MRT), S.P. Sit'ko, ed., Vidguk, Kiev, 1992, in Russian; In Methodological Instructions for Physicians in Using Microwave Resonance Therapy, V.D.Zhukovskiy, ed., GPK, Moscow, 1996, in Russian.

34. Sit'ko, S.P., Andreyev, Ye.A. and Dobronravova, I.S.: The whole as a result of selforganization, Journal of Biological Physics 16: 71-73, 1988; Sit'ko S.P. and Gizhko, V.V.: Towards a quantum physics of the living state, Journal of Biological Physics 18: 1-10, 1991; Gizhko V.V. and Sit'ko, S.P.: Coherent microwave electromagnetic fields as a physical model of macroscopic quantum states of the multicellular organism, Journal of Physics of the Alive 1: 103-109, 1993; Sit'ko, S.P.: Quantum physics of the alive: Medical aspects, in Proceedings of ECPD Workshop Brain \& Consciousness, Lj. Rakić, G. Kostopoulos, D. Raković and Dj. Koruga, eds., Belgrade, 1997.

35. Fröhlich, H.: Long-range coherence and energy storage in biological systems, International Journal of Quantum Chemistry 2: 641-649, 1968; Fröhlich, H.: Theoretical physics and biology, in Biological Coherence and Response to External Stimuli, H. Fröhlich, ed., Springer, New York, 1988.

36. Hopfield, J.J.: Neural networks and physical systems with emergent collective computational abilities, Proceedings of National Academy of Science USA 79: 2554-2558, 1982; Haken, H.: Synergetic Computers and Cognition (A Top-Down Approach to Neural Nets), Springer, Berlin, 1991.

37. Such a picture is very close to those of associative neural networks [36,38] in their energyconfiguration spaces, and to pattern recognition as convergence of the neural networks to the bottoms of the potential hypersurfaces, being the attractors of neural networks memory patterns (cf. Fig. 1). This similarity of the quantum and neural network pictures might not be only superficial, as (real-valued) mathematical formalism of Hopfield's and Haken's associative neural network models [36] is analogical [38] to the (complex-valued) mathematical formalism of Feynman's propagator version of the Schrödinger equation [39]. This also support the EM/ionic "optical" MW/ULF neural network-like function of the acupuncture system, and its essential relation to (complex-valued quantum relativistic) consciousness, as strongly suggested from modeling of altered states of consciousness [9,10] (cf. also footnote 1); in this framework, (complex-valued) EM/ionic "optical" MW/ULF acupuncture quantum neural network (like oscillatory holographic Hopfield-like neural networks [38]) triggers the "collapse" of (complexvalued) quantum wave-function - via relativistic wormhole (real-valued) classical environment in transitional states of consciousness [40] (cf. also [16]) - and thus transform the (complexvalued) probabilistic dynamics of quantum world, related to (complex-valued) consciousness, into the (real-valued) deterministic dynamics of classical world, related to (real-valued) biological neural networks [38] - which might offer biophysical prospects for hypothetical conscious brain-like computers [41]. In support to this, there are strong indications [42] that 
biological neural networks essentially cooperate with quantum networks in the brain.

38. Peruš, M.: Neuro-quantum parallelism in mind-brain and computers, Informatica 20: 173-183, 1996; Peruš, M.: Multi-level synergetic computation in brain, Advances in Synergetics 9, 1998, in press; and references therein.

39. Feynman R.P. and Hibbs, A.R.: Quantum Mechanics and Path Integrals, McGraw-Hill, New York, 1965; Bjorken J.D. and Drell, S.D.: Relativistic Quantum Fields, McGraw-Hill, New York, 1965, Ch. 6: Propagator Theory.

40. Raković, D.: Consciousness and quantum collapse: Biophysics versus relativity, The Noetic Journal 1: 34-41, 1997.

41. Raković, D.: Prospects for conscious brain-like computers: Biophysical arguments, Informatica 21: 507-516, 1997.

42. Pribram, K.H.: Brain and Perception, Lawrence Erlbaum A., Hillsdale, 1991; in Rethinking Neural Networks (Quantum Fields and Biological Data), K.H. Pribram, ed., Lawrence Erlbaum A., Hillsdale, 1993.

43. Unfortunately, only non complete but extended list of over twenty relevant original references from 1969 to 1984 (on Soviet authors and years of publications, but not on the original journals) is cited in Ref. 31.

44. Jovanović-Ignjatić, Z.: Microwave resonance therapy: New therapeutic possibilities, in Exogenous and Endogenous Biological Interactions: Biophysical and Biochemical Aspects, D. Raković and Dj. Koruga, eds., Molecular Machines Research Center \& IHIS FST Center, Belgrade, 1998, in Serbian.

45. Jovanov, E., Raković, D., Radivojević, V., Kušić, D., Šuković, P. and Car, M.: Software support for monitoring spatio-temporal EEG changes: An example of evaluation of state of consciousness, ISCA Conf. Comput. Med., Lafayette, 1995; Jovanov, E., Raković, D., Radivojević, V. and Kušić, D.: Band power envelope analysis - A new method in quantitative EEG, Proc. $17^{\text {th }}$ Ann. Int. Conf. IEEE/EMBS, Montreal, 1995; Radenović, D., Raković, D., Jovanović-Ignjatić, Z., Tomašević, M., Radivojević, V. and Jovanov, E.: Relaxation induced by microwave resonance therapy: EEG correlates, Proc. ECPD Symp. Brain \& Consciousness, Belgrade, 1997; Šuković, P., Radivojević, V., Martinović, Ž., Raković, D. and Jovanov, E.: A novel neural network approach to estimation of vigilance level from EEG power spectrum, ibid; Tomašević, M., Raković, D., Jovanov, E., Radivojević, V. and Car, M.: EEG correlates of Transcendental Meditation, ibid; Škarić, L., Tomašević, M., Raković, D., Jovanov, E., Radivojević, V., Šuković, P., Car, M. and Radenović, D.: EEG correlates of musicogenic states of consciousness, ibid; Tomašević, M., Jovanov, E., Raković, D., Šuković, P., Stanojlović, S. and Car, M.: EEG correlates of healer/healee states of consciousness, ibid; Raković, D., Tomašević, M., Jovanov, E., Radivojević, V., Šuković, P., Martinović, Ž., Car, M., Radenović, D., Jovanović-Ignjatić, Z. and Škarić, L.: Electroencephalographic (EEG) correlates of some activities which may alter consciousness: The transcendental meditation technique, musicogenic states, microwave resonance relaxation, healer/healee interaction, and alertness/ drowsiness, Int. Conf. Consciousness in Science \& Philosophy, Charleeston, IL, 1998, to be published in Informatica.

46. Nader, T.: Human Physiology: Expression of Veda and the Vedic Literature, Maharishi Vedic Univ., Vlodrop, The Netherlands, 1995.

47. Wallace, R.K.: The Physiology of Consciousness, Maharishi Int. Univ., Fairfield, USA, 1993; Wallace, R.K.: Physiological effects of transcendental meditation, Science 167: 1751-1754, 1970; in Scientific Research of the Transcendental Meditation Program - Collected Papers, D.W. Orme-Johnson and J. T. Farrow, eds., MERU Press, Seelisberg, Switzerland, Vol. 1, 1977.

48. Banquet, J.P.: Spectral Analyses of the EEG in meditation, EEG Clin. Neurophysiol. 35: 143$151,1973$.

49. Levine, P.H., Hebert, J.R., Haynes, C.T. and Strobel, U.: EEG coherence during the transcendental meditation technique, in Scientific Research of the Transcendental Meditation 
Program - Collected Papers, D.W. Orme-Johnson and J.T. Farrow, eds., MERU Press, Seelisberg, Switzerland, Vol. 1, 187-207, 1977.

50. Kras, D.J.: The transcendental meditation technique and EEG alpha activity, ibid., 173-181.

51. Rouzeré, A.M., Badawi, K. and Hartmann, R.: High Amplitude Fronto-central Alpha and Theta Activity During the Transcendental Meditation Technique, Department of Neurophysiology, MERU, Seelisberg, Switzerland, 1979.

52. Banquet J.P. and Sailhan, M.: EEG analysis of spontaneous and induced states of consciousness, in Scientific Research of the Transcendental Meditation Program - Collected Papers, D.W. Orme-Johnson and J.T. Farrow, eds., MERU Press, Seelisberg, Switzerland, Vol. 1, 165-172, 1977, in English, and Revue d'Electroencephalographie et Neurophysiol. Clinique 4: 445-453, 1974, in French.

53. Mundy-Castle, A.C.: The electroencephalogram and mental activity, EEG Clin. Neurophysiol. 9: 643-655, 1957.

54. Forem, J.: Transcendental Meditation: Maharishi Mahesh Yogi and the Science of Creative Intelligence, Dutton, New York, NY, 1973.

55. Alexander, N. and Larimore, W.E.: Distinguishing Between Transcendental Meditation and Sleep According to Electrophysiological Criteria, Department of Psychology and Social Relations, Harvard University, Cambridge, MA, and The Analytic Sciences Corporation, Reading, MA, 1981.

56. Davidson, J.M.: The physiology of meditation and mystical states of consciousness, in Meditation: Classic and Contemporary Perspectives, D.H. Shapiro and R.N. Walsh, eds., ADLINE Publishing Company, New York, 376-395, 1984.

57. Lyubimov N.N. and Lyubimov, S.N.: Dual reactivity of cerebrum during application of the special form of psychological training - transcendental meditation, ECPD Workshop Brain and Consciousness '98, June 24-25, Belgrade.

58. Ellias S.A. and Grossberg, S.: Pattern information, contrast control, and oscillations in the shortterm memory of shunting on-center of surround networks, Biological Cybernetics 20: 69-98, 1975.

59. Roederer, J.G.: Physical and neuropsychological foundations of music: The basic questions, in Music, Mind, and Brain: The Neuropsychology of Music, M. Clynes, ed., Plenum, New York, 1982, and references therein.

60. Baars, B.J.: A Cognitive Theory of Consciousness, Cambridge Univ., Cambridge MA, 1988.

61. Rouget, G.: La Musique et la Transe, Gallimard, Paris, 1980; Jourdain, R.: Music, the Brain, and Ecstasy, Avon Books, New York, 1997.

62. Raković, D. and Dugić, M.: Consciousness mediated quantum gravitational collapse via generated wormholes: From macroscopic biophysical to microscopic quantum arguments, in Proc. Joint Conf. Inform. Sci./Vol. 2: $3^{\text {rd }}$ Int. Conf. Comput. Intell. \& Neurosci, P. P. Wang and G. Georgiou, ed., Assoc. for Intelligent Machinery, RTP, NC, 265-268, 1998.

63. Monroe, R.: Journeys Out of the Body, Doubleday, Garden City, NY, 1971.

64. Kaznacheev, V.P. and Trofimov, A.V.: Cosmic Consciousness of Humanity, Problems of New Cosmogony, Elendis - Progress, Tomsk, Russia, 1992.

65. Broughton, R.: Human consciousness and sleep/waking rhythms, in Handbook of States of Consciousness, B.B. Wolman and M. Ullman, eds., Van Nostrand Reinhold, New York, 1986.

66. In A Manual of Standardized Terminology, Techniques and Scoring System for Sleep Stages of Human Subjects, A. Rechtschaffen and A. Kales, eds., U.S. Public Health Service, U.S. Government Printing Office, Washington DC, 1968.

67. Ilanković, N., Ilanković, V. and Jašović-Gašić, M.: Sleep Disorder - Diagnostics and Curing, Cibif, Belgrade, 1995, in Serbian.

68. Hasan, J.: Past and future of computer-assisted sleep analysis and drowsiness assessment, J. Clin. Neurophysiol. 13: 295-313, 1996.

69. Basar, E.: EEG Brain Dynamics, Elsevier, Amsterdam, 1980. 
70. Wright, K.P., Badio, P. and Wauquier, A.: Topographical and temporal patterns of brain activity during the transition from wakefulness to sleep, Sleep 18: 880-889, 1995.

71. Jung, T., Makeig, S., Stensmo, M. and Sejnowski, T. J.: Estimating alertness from the EEG power spectrum, IEEE Trans. BME 44, 1997.

72. Matoušek, M. and Petersén, I.: A method assessing alertness fluctuations from EEG spectra, EEG Clin. Neurophysiol. 55: 108-113, 1983.

73. Belyavin, A. and Wright, N.: Changes in electrical activity of the brain with the vigilance, EEG Clin. Neurophysiol. 66: 137-144, 1987.

74. Nakamura, M., Sugi, T., Ikeda, A., Kakigi, R. and Shibasaki, H.: Clinical application of automatic integrative interpretation of awake background EEG: Quantitative interpretation, report making, and detection of artifacts and reduced vigilance level, EEG Clin. Neurophysiol. 98: 103-112, 1996.

75. Streitberg, B., Röhmel, J., Herrmann, W.M. and Kubicki, S.: COMSTAT rule for vigilance classification based on spontaneous EEG activity, Neuropsychobiology 17: 105-117, 1987.

76. Cajochen, C., Brunner, D.P., Kräuchi, K., Graw, P. and Wirz-Justice, A.: Power density in theta/alpha frequencies of the waking EEG progressively increases during sustained wakefulness, Sleep 18: 890-894, 1995.

77. MATLAB 4.0 User's Guide, The MathWorks Inc., Natick, USA, 1994. 